\title{
Supporting Information 1: Synthesis and Physical Study of Perylene and Anthracene Polynitrile as Electron Acceptors
}

Ting-Yu Li, Yi-Chun Lin, Yu-Huei Song, Hsiu-Feng Lu, Ito Chao, ${ }^{*}$ Chih-Hsiu Lin* Institute of Chemistry, Academia Sinica, Academia Road, Sec. 2, No. 128, Taipei, Taiwan, Republic of China

\section{Synthetic Procedures and UV, Fluorescence, CV Data}

Synthetic procedures for 2-9 .p2-15

Synthetic procedures for $\mathbf{1 1 - 1 7}$ p16-25

UV monitoring of 16-17 transformation. .p26

UV, fluorescence spectrum and CV data of 5-9 p27-30 
Materials and general procedures: All starting compounds and reagents were purchased from commercial sources and used without further purification. All reaction solvents were distilled or passed through a column of activated alumina before use. All reactions were performed under 1 atmosphere of dried nitrogen in dried reaction vessels and well mixed with magnetic stirring devices. All reactions were monitored by analytical thin layer chromatography (TLC, 250 EM silica gel 60 $\mathrm{F}_{254}$ plates, visualized with a $254 \mathrm{~nm}$ UV lamp) or ${ }^{1} \mathrm{H}$ NMR. HPLC grade solvents were used for extraction and chromatography. Flash column chromatography was performed with Merck silica gel $60(1.11567 .9025,0.040-0.063 \mathrm{~mm})$ as the stationary phase. All reported ratios of eluent solvents mixtures are based on volume.

General protocols for photophysical and electrochemical studies: The absorption and emission spectrum were recorded in dichloromethane solution $\left(\sim 10^{-5} \mathrm{M}\right)$. The fluorescent quantum yields were measured with coumarin 6 as the reference. The cyclic voltammetry studies were performed in dichloromethane solution $\left(\sim 10^{-4} \mathrm{M}\right)$ with n-Bu $\mathrm{NPF}_{6}$ as the supporting electrolyte. $\mathrm{Ag} / \mathrm{AgNO}_{3}$ is the reference electrode and the scan rate was set to $50 \mathrm{mV} / \mathrm{sec}$. The LUMO energy levels of electron acceptors were estimated with ferrocene $(\mathrm{HOMO}=-4.80 \mathrm{eV})$ as the standard. The HOMO energy levels were calculated from optical gaps and LUMO levels (HOMO = LUMO-Egap). 

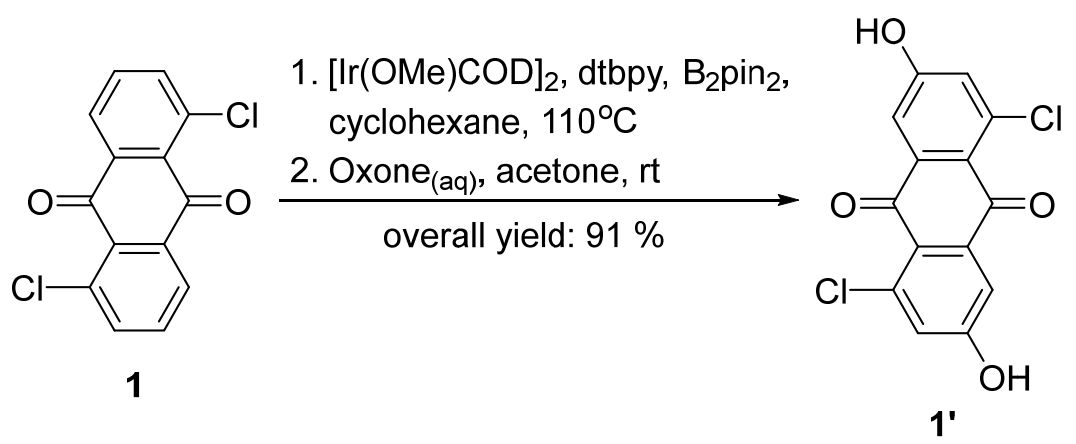

1,5-dichloro-3,7-dihydroxyanthraquinone

(1') Under inert atmosphere,1,5-dicloroanthraquinone (1, $100 \mathrm{mg}, 0.360 \mathrm{mmol}),(1,5-$ cyclooctadiene)(methoxy)iridium(I) dimer (29 mg, $0.043 \mathrm{mmol}$ ), 4,4'-ditert-butyl-2,2'-dipyridyl (24 mg, $0.087 \mathrm{mmol})$ and bis(pinacolato)diboron (275 mg, $1.083 \mathrm{mmole})$ were dissolved in cyclohexane $(10 \mathrm{~mL})$. The sealed tube was heated at $110{ }^{\circ} \mathrm{C}$ for $12 \mathrm{hr}$ before cooled to room temperature. The brownish precipitation was collected and washed with cold hexane $(50 \mathrm{~mL})$ before dried in vacuo. The anthraquinone bis(boronate) was used in the next step without further purification.

An aqueous solution of oxone ( $887 \mathrm{mg}, 1.443 \mathrm{mmol}$ in $5 \mathrm{~mL}$ water) was added to an acetone solution of the crude anthraquinone bis(boronate) (crude product in $100 \mathrm{~mL}$ ). The oxidation proceeded at room temperature overnight. The yellow precipitation was collected and washed with water and ether (100 $\mathrm{mL}$ each). The product was then dried in vacuo to give 1' as a yellow solid (101 $\mathrm{mg}, 91 \%)$. 
${ }^{1} \mathrm{H}$ NMR (400 MHz, DMSO-d 6 ) $\delta$ (ppm): 7.46 (s, 2H), 7.15 (s, 2H). ${ }^{13} \mathrm{C}$ NMR (100 MHz, DMSO-d 6 ) $\delta(\mathrm{ppm}): 178.7,162.2,138.7,135.2,122.9$, 120.4, 113.1. HRMS (EI) m/e: calcd for $\mathrm{C}_{14} \mathrm{H}_{6} \mathrm{O}_{4} \mathrm{Cl}_{2}[\mathrm{M}]^{+}: 307.9643$ found: 307.9637. IR $v\left(\mathrm{~cm}^{-1}\right): 3400,1643,1311,1088,751$.
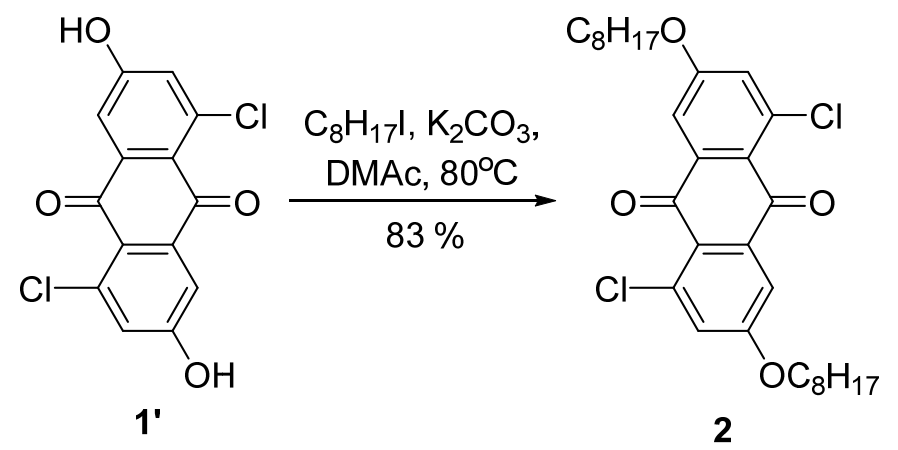

1,5-dichloro-3,7-bis(octyloxy)anthraquinone (2) Under inert atmosphere, 1' (714 mg, $2.309 \mathrm{mmol})$ and potassium carbonate (1120 mg, $8.085 \mathrm{mmol}$ ) were placed in a round bottom flask. To this reaction vessel were then added $N, N$-dimethylacetamide $(12 \mathrm{~mL})$ and 1-iodooctane $(1.05$ $\mathrm{mL}, 5.775 \mathrm{mmol})$. The reaction was stirred at $80{ }^{\circ} \mathrm{C}$ for $8 \mathrm{hr}$. The solvent was removed in vacuo. The residue was dissolved in $\mathrm{CH}_{2} \mathrm{Cl}_{2}(200$ $\mathrm{mL})$ and the solution was washed with water $(100 \mathrm{~mL} \times 3)$. The organic phase was dried over $\mathrm{MgSO}_{4}$ and concentrated. The white solid thus obtained was further washed with ether $(200 \mathrm{~mL})$ before dried in vacuo to furnish the bis(octyloxy)anthraquinone product 2 as white powder (1022 mg, $83 \%)$. 
${ }^{1} \mathrm{H}$ NMR (400 MHz, $\left.\mathrm{CDCl}_{3}\right) \delta(\mathrm{ppm}): 7.62(\mathrm{~d}, J=2.4 \mathrm{~Hz}, 2 \mathrm{H}), 7.12$ (d, $J=2.4 \mathrm{~Hz}, 2 \mathrm{H}), 4.07$ (t, $J=6.4 \mathrm{~Hz}, 4 \mathrm{H}), 1.79$ (quin, $J=6.4 \mathrm{~Hz}, 4 \mathrm{H}$ ), $1.47-1.40(\mathrm{~m}, 4 \mathrm{H}), 1.32-1.26(\mathrm{~m}, 16 \mathrm{H}), 0.86(\mathrm{t}, J=6.4 \mathrm{~Hz}, 6 \mathrm{H}) .{ }^{13} \mathrm{C} \mathrm{NMR}$ $\left(100 \mathrm{MHz}, \mathrm{CDCl}_{3}\right) \delta(\mathrm{ppm}): 179.4,162.8,138.7,136.4,123.4,121.9,111.4$ 69.1, 31.7, 29.2, 29.1, 28.8, 25.8, 22.6, 14.0. HRMS (FAB) m/e: calcd for $\mathrm{C}_{30} \mathrm{H}_{39} \mathrm{O}_{4} \mathrm{Cl}_{2}[\mathrm{M}+\mathrm{H}]^{+}: 533.2225$ found: 533.2224. IR $v\left(\mathrm{~cm}^{-1}\right)$ : 2923, 1636, $1304,1092,703 . \mathrm{Mp}=89^{\circ} \mathrm{C}$

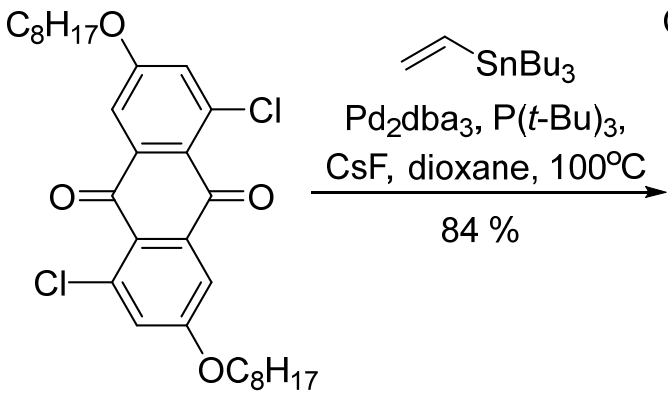

2

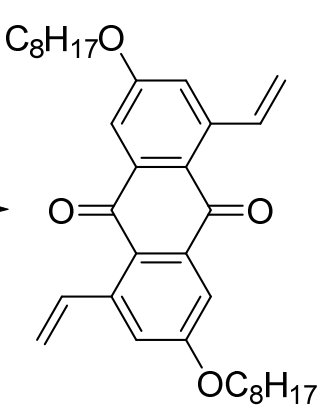

3

3,7-bis(octyloxy)-1,5-divinylanthraquinone (3) Under inert atmosphere, compound 3 (150 mg, $0.281 \mathrm{mmol}$ ), tri-tert-butylphosphine (7 mg, 0.034 mmol), tris(dibenzylideneacetone)dipalladium ( $8 \mathrm{mg}, 0.008 \mathrm{mmol})$, and cesium fluoride (188 mg, $1.237 \mathrm{mmol})$ were dissolved in dioxane $(5 \mathrm{~mL})$ in a pressurized tube. To the solution was then added tributyl vinyltin $(0.17 \mathrm{~mL}, 0.590 \mathrm{mmol})$. The reaction vessel was sealed and heated to 100 ${ }^{\circ} \mathrm{C}$ for $8 \mathrm{hr}$. The solvent was removed in vacuo and the residue is 
dissolved in $\mathrm{CH}_{2} \mathrm{Cl}_{2}(50 \mathrm{~mL})$. The solution was passed through a celite pad before washed with water $(20 \mathrm{~mL} \times 3)$. The organic phase was dried over $\mathrm{MgSO}_{4}$ and concentrated to give the crude product. Pure 3 was obtained as a yellow solid (122 mg, $84 \%$ ) after flash chromatography $\left(\mathrm{SiO}_{2}, \mathrm{CH}_{2} \mathrm{Cl}_{2} /\right.$ hexane, $\left.30 \%\right)$.

${ }^{1} \mathrm{H}$ NMR (400 MHz, $\left.\mathrm{CDCl}_{3}\right) \delta(\mathrm{ppm}): 7.66(\mathrm{dd}, J=17.2,10.8 \mathrm{~Hz}, 2 \mathrm{H})$, $7.53(\mathrm{~d}, J=2.4 \mathrm{~Hz}, 2 \mathrm{H}), 7.06(\mathrm{~d}, J=2.4 \mathrm{~Hz}, 2 \mathrm{H}), 5.53(\mathrm{~d}, J=17.6 \mathrm{~Hz}, 2 \mathrm{H})$, $5.35(\mathrm{~d}, J=11.6 \mathrm{~Hz}, 2 \mathrm{H}), 4.02(\mathrm{t}, J=6.8 \mathrm{~Hz}, 4 \mathrm{H}), 1.77$ (quin, $J=6.8 \mathrm{~Hz}$, 4H), $1.43-1.39(\mathrm{~m}, 4 \mathrm{H}), 1.32-1.26(\mathrm{~m}, 16 \mathrm{H}), 0.86(\mathrm{t}, J=6.8 \mathrm{~Hz}, 6 \mathrm{H}) .{ }^{13} \mathrm{C}$ NMR (100 MHz, $\left.\mathrm{CDCl}_{3}\right) \delta(\mathrm{ppm}): 183.2,162.7,143.3,138.0,137.4,122.7$, $119.8,116.7,110.9,68.5,31.7,29.3,29.1,29.0,25.9,22.6,14.0$. HRMS (FAB) m/e: calcd for $\mathrm{C}_{34} \mathrm{H}_{45} \mathrm{O}_{4}[\mathrm{M}+\mathrm{H}]^{+}: 517.3318$ found: 517.3317 . IR $v$ $\left(\mathrm{cm}^{-1}\right): 3082,2926,1660,1589,1556,1304,1134,737$.

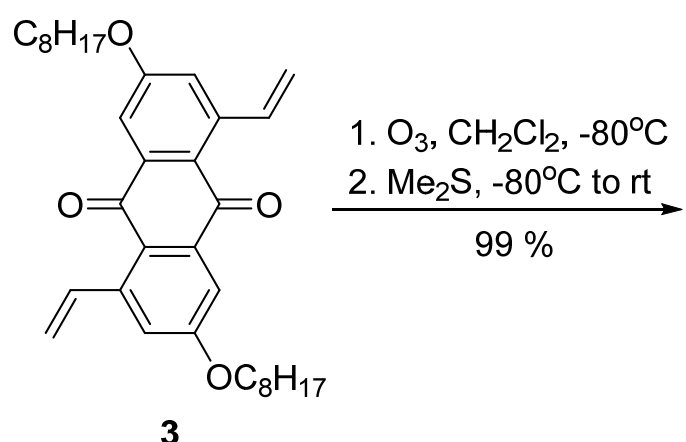

3

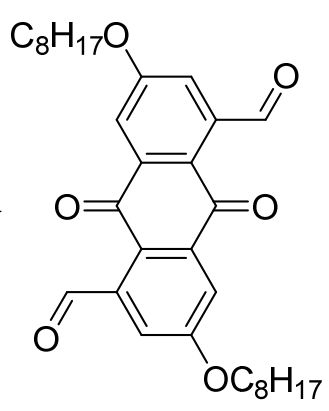

4 
3,7-bis(octyloxy)-1,5-dicarbaldehyde-anthraquinone (4) In a twoneck round bottom flask was placed a solution of $\mathbf{3}(100 \mathrm{mg}, 0.193 \mathrm{mmol})$ in $\mathrm{CH}_{2} \mathrm{Cl}_{2}(100 \mathrm{~mL})$. After the system was chilled to $-80{ }^{\circ} \mathrm{C}$, ozone was bubbled in for $5 \mathrm{~min}$. Dimethyl sulfide $(0.07 \mathrm{~mL}, 0.968 \mathrm{mmol})$ was added and the reaction was warmed back to room temperature. The reaction solution was washed with water $(100 \mathrm{~mL} \times 3)$. The organic layer was dried over $\mathrm{MgSO}_{4}$, concentrated in vacuo to give a grey solid. The crude product was washed with ether $(100 \mathrm{~mL})$ before dried in vacuo to give 4 as a yellow solid (100 mg, $99 \%)$.

${ }^{1} \mathrm{H}$ NMR (400 MHz, $\left.\mathrm{CDCl}_{3}\right) \delta(\mathrm{ppm}): 10.78$ (s, 2H), 7.87 (d, $J=2.8 \mathrm{~Hz}$, 2H), $7.53(\mathrm{~d}, J=2.8 \mathrm{~Hz}, 2 \mathrm{H}), 4.16(\mathrm{t}, J=6.8 \mathrm{~Hz}, 4 \mathrm{H}), 1.84$ (quin, $J=6.8$ $\mathrm{Hz}, 4 \mathrm{H}), 1.47$ (quin, $J=6.8 \mathrm{~Hz}, 4 \mathrm{H}), 1.34-1.28(\mathrm{~m}, 16 \mathrm{H}), 0.88(\mathrm{t}, J=6.8$ $\mathrm{Hz}, 6 \mathrm{H}) .{ }^{13} \mathrm{C} \mathrm{NMR}\left(100 \mathrm{MHz}, \mathrm{CDCl}_{3}\right) \delta(\mathrm{ppm}): 192.2,182.5,163.8,141.4$ $136.9,125.3,119.6,115.4,69.4,31.8,29.2,29.2,28.9,25.8,22.6,14.0$. HRMS (FAB) m/e: calcd for $\mathrm{C}_{32} \mathrm{H}_{41} \mathrm{O}_{6}[\mathrm{M}+\mathrm{H}]^{+}: 521.2903$ found: 521.2909 . IR $v\left(\mathrm{~cm}^{-1}\right): 3074,2953,2914,2853,1682,1655,1587,1562,1304,1260$, $1125,715$. 

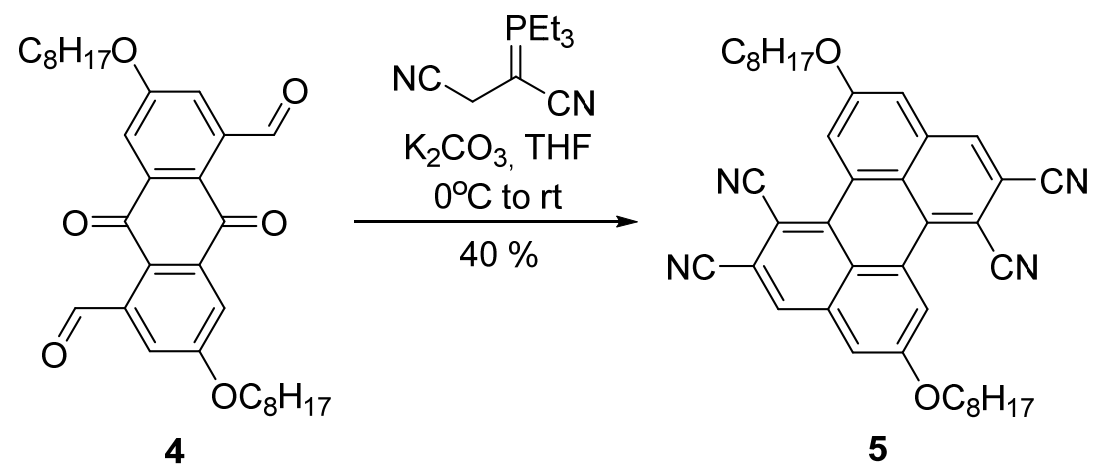

5,11-bis(octyloxy)perylene-1,2,7,8-tetracarbonitrile (5) The Wittig reagent was prepared according to following procedure. Under inert atmosphere, fumaronitrile (375 $\mathrm{mg}, 4.801 \mathrm{mmol}$ ) was dissolved in THF $(10 \mathrm{~mL})$ and to this solution was added a THF solution of triethylphosphine (1 M, $4.8 \mathrm{~mL}, 4.80 \mathrm{mmol})$. The mixture was refluxed overnight.

In another flask, compound $4(1000 \mathrm{mg}, 1.920 \mathrm{mmol})$ and potassium carbonate (1327 mg, $9.603 \mathrm{mmol})$ was suspended in THF $(96 \mathrm{~mL})$ at $0{ }^{\circ} \mathrm{C}$. To this mixture was slowly added the Wittig reagent solution prepared previously. The reaction was stirred at room temperature for $24 \mathrm{~h}$. The volatiles were removed in vacuo. The residue was dissolved in $\mathrm{CH}_{2} \mathrm{Cl}_{2}$ $(500 \mathrm{~mL})$ and the solution was washed with water $(200 \mathrm{~mL} \times 3)$. The organic phase was dried over $\mathrm{MgSO}_{4}$ and concentrated. The dark crude product was purified by flash chromatography $\left(\mathrm{SiO}_{2} ; \mathrm{CH}_{2} \mathrm{Cl}_{2} /\right.$ hexane, $\left.50 \%\right)$ to furnish perylene product 5 as a yellow powder (468 mg, $40 \%$ ).

${ }^{1} \mathrm{H}$ NMR (500 MHz, $\left.\mathrm{CDCl}_{3}\right) \delta(\mathrm{ppm}): 8.79(\mathrm{~d}, J=1.5 \mathrm{~Hz}, 2 \mathrm{H}), 8.12$ 
(s, 2H), $7.30(\mathrm{~d}, J=1.5 \mathrm{~Hz}, 2 \mathrm{H}), 4.22(\mathrm{t}, J=6.5 \mathrm{~Hz}, 4 \mathrm{H}), 1.93$ (quin, $J=$

$6.5 \mathrm{~Hz}, 4 \mathrm{H}), 1.55-1.49(\mathrm{~m}, 4 \mathrm{H}), 1.42-1.29(\mathrm{~m}, 16 \mathrm{H}), 0.88$ (t, $J=6.5 \mathrm{~Hz}$ 6H). $\left.{ }^{13} \mathrm{C} \mathrm{NMR} \mathrm{(125} \mathrm{MHz,} \mathrm{CDCl}_{3}\right) \delta(\mathrm{ppm}): 160.5,135.4,135.0,134.0$, 129.3, 124.5, 122.1, 117.7, 115.8, 115.2, 111.3, 103.6, 69.5, 31.8, 29.3, 29.2, 28.9, 25.9, 22.6, 14.1. HRMS (FAB) $\mathrm{m} / \mathrm{e}$ : calcd for $\mathrm{C}_{40} \mathrm{H}_{40} \mathrm{O}_{2} \mathrm{~N}_{4}\left[\mathrm{M}^{+}\right]$: 608.3151 found: $608.3160 . \operatorname{IR} \vee\left(\mathrm{cm}^{-1}\right): 3059,2917,2235(\mathrm{C} \equiv \mathrm{N}$ stretch), $2211(\mathrm{C} \equiv \mathrm{N}$ stretch $), 1612,1434,1375,1191 . \mathrm{UV} / \mathrm{Vis}\left(\mathrm{CH}_{2} \mathrm{Cl}_{2}\right): \lambda_{\max }=432$, $458 \mathrm{~nm}$. Fluorescence $\left(\mathrm{CH}_{2} \mathrm{Cl}_{2}, \lambda_{\mathrm{ex}}=458 \mathrm{~nm}\right): \lambda_{\mathrm{em}}=500 \mathrm{~nm} . \Phi=0.49$.

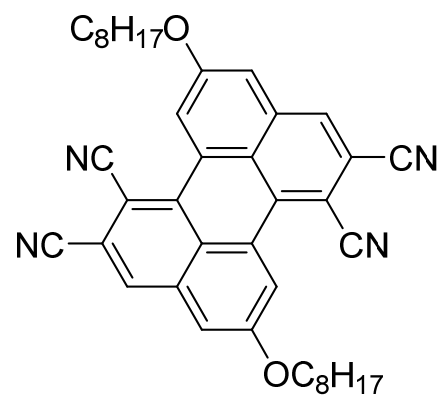

5

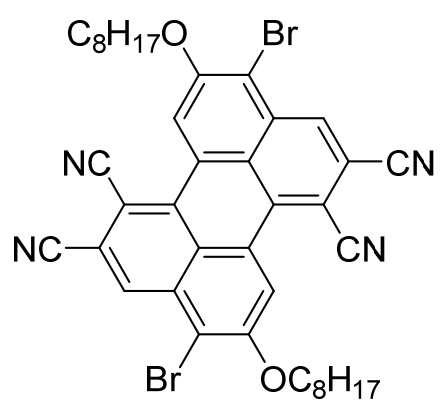

$\mathbf{5}^{\prime}$

4,10-dibromo-5,11-bis(octyloxy)perylene-1,2,7,8-tetracarbonitrile

Under inert atmosphere, compound 5 (20 mg, $0.033 \mathrm{mmol})$ was dissolved in a mixture of $\mathrm{CHCl}_{3}(1.5 \mathrm{~mL})$ and trifluoroacetic acid $(1.5 \mathrm{~mL}) . \mathrm{N}$ Bromosuccinimide (30 $\mathrm{mg}, 0.164 \mathrm{mmol}$ ) was added and the reaction was stirred at room temperature for $48 \mathrm{hr}$. Volatiles were removed in vacuo. The reside was dissolved in $\mathrm{CH}_{2} \mathrm{Cl}_{2}(50 \mathrm{~mL})$ and the solution was washed 
with $\mathrm{Na}_{2} \mathrm{SO}_{3(\mathrm{aq})}\left(20 \mathrm{~mL}\right.$ x 3). The organic phase was dried over $\mathrm{MgSO}_{4}$ before concentrated to give brominated $\mathbf{5}^{\prime}$ as an orange power (25 mg, 99 $\%) \circ$

${ }^{1} \mathrm{H}$ NMR (400 MHz, $\left.\mathrm{CDCl}_{3}\right) \delta(\mathrm{ppm}): 9.03(\mathrm{~s}, 1 \mathrm{H}), 8.78(\mathrm{~s}, 1 \mathrm{H}), 4.43(\mathrm{t}, J$ $=6.4 \mathrm{~Hz}, 4 \mathrm{H}), 1.97$ (quin, $J=6.4 \mathrm{~Hz}, 4 \mathrm{H}), 1.40-1.24(\mathrm{~m}, 20 \mathrm{H}), 0.88(\mathrm{t}, J$ $=6.4 \mathrm{~Hz}, 6 \mathrm{H}) .{ }^{13} \mathrm{C} \mathrm{NMR}\left(100 \mathrm{MHz}, \mathrm{CDCl}_{3}\right) \delta(\mathrm{ppm}): 157.2,135.5,134.1$, $133.9,128.0,124.9,117.8,117.7,116.2,115.6,113.7,104.5,71.1,31.8$, 29.2, 29.2, 29.1, 25.9, 22.6, 14.1 HRMS (EI) m/e: calcd for $\mathrm{C}_{40} \mathrm{H}_{38} \mathrm{O}_{2} \mathrm{~N}_{4} \mathrm{Br}_{2}$ [M+]: 764.1361 found: 764.1357. IR $\vee\left(\mathrm{cm}^{-1}\right): 3053,2928,2223(\mathrm{C} \equiv \mathrm{N}$ stretch), 1597, 1422, 1377, 1119, 516. $\mathrm{Mp}=217^{\circ} \mathrm{C}$ (decomp. $)$
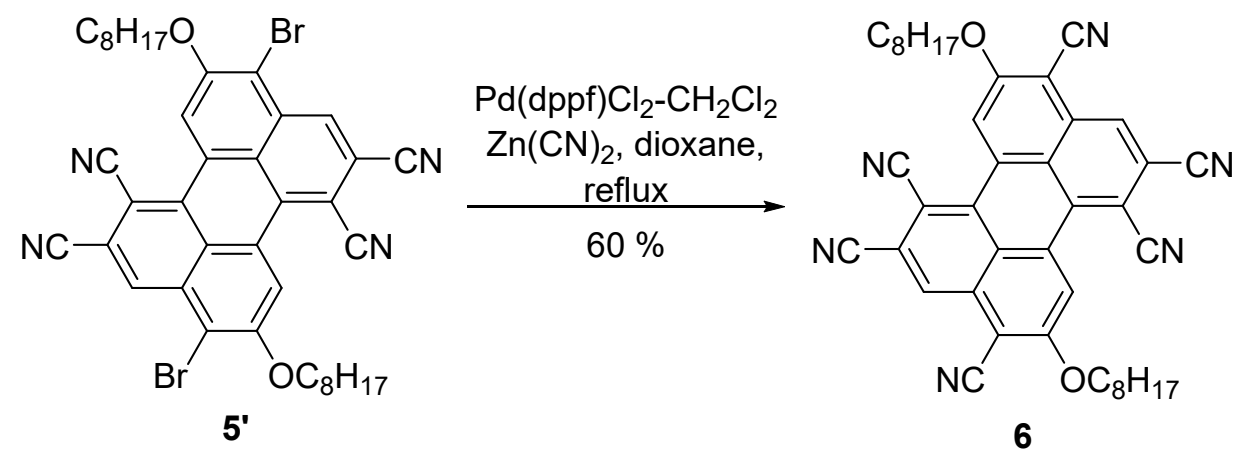

5,11-bis(octyloxy)perylene-1,2,4,7,8,10-hexacarbonitrile (6) Under inert atmosphere, compound 5' (105 mg, $0.137 \mathrm{mmol}), \quad\left[1,1^{\prime}-\right.$ bis(diphenylphosphino)ferrocene]dichloropalladium(II) dichloromethane adduct (11 mg, $0.014 \mathrm{mmol})$ and zinc cyanide (49 mg, $0.411 \mathrm{mmol})$ were 
placed in a pressurized tube. Dioxane $(3 \mathrm{~mL})$ were added and the sealed tube was heated at $120^{\circ} \mathrm{C}$ overnight. The solvent was removed in vacuo and the residue was dissolved in $\mathrm{CH}_{2} \mathrm{Cl}_{2}(100 \mathrm{~mL})$. The solution was filtered through a celite pad and the solution was washed with water (50 $\mathrm{mL}$ x 3). The organic phase was dried over $\mathrm{MgSO}_{4}$ and concentrated. The dark crude product was purified by flash chromatography $\left(\mathrm{SiO}_{2}\right.$; $\mathrm{CH}_{2} \mathrm{Cl}_{2} /$ hexane, $70 \%$ ) to give 6 as a red solid (54 mg, $60 \%$ ).

${ }^{1} \mathrm{H}$ NMR (500 MHz, $\left.\mathrm{CDCl}_{3}\right) \delta(\mathrm{ppm}): 9.17$ (s, 1H), 8.67 (s, 1H), 4.50 (t, $J$ $=6.5 \mathrm{~Hz}, 4 \mathrm{H}), 2.02-1.97(\mathrm{~m}, 4 \mathrm{H}), 1.59-1.53(\mathrm{~m}, 4 \mathrm{H}), 1.42-1.23(\mathrm{~m}, 16 \mathrm{H})$, $0.87(\mathrm{t}, J=6.5 \mathrm{~Hz}, 6 \mathrm{H}) .{ }^{13} \mathrm{C} \mathrm{NMR}\left(125 \mathrm{MHz}, \mathrm{CDCl}_{3}\right) \delta(\mathrm{ppm}): 163.4,134.9$, 134.3, 132.4, 132.3, 123.6, 117.8, 117.5, 117.0, 114.6, 112.8, 106.7, 99.3, 71.7, 31.7, 29.1, 29.1, 28.9, 25.7, 22.6, 14.1. HRMS (FAB) m/e: calcd for $\mathrm{C}_{42} \mathrm{H}_{39} \mathrm{O}_{2} \mathrm{~N}_{6}[\mathrm{M}+\mathrm{H}]^{+}: 659.3134$ found: 659.3130. IR $v\left(\mathrm{~cm}^{-1}\right)$ : 3054, 2927, $2223(\mathrm{C} \equiv \mathrm{N}$ stretch $), 1634,1597,1422,1377,1118 . \mathrm{UV} / \mathrm{Vis}\left(\mathrm{CH}_{2} \mathrm{Cl}_{2}\right)$ : $\lambda_{\max }=458,490 \mathrm{~nm}$. Fluorescence $\left(\mathrm{CH}_{2} \mathrm{Cl}_{2}, \lambda_{\mathrm{ex}}=490 \mathrm{~nm}\right): \lambda_{\mathrm{em}}=510,540 \mathrm{~nm}$. $\Phi=0.39 . \quad \mathrm{Mp}=278^{\circ} \mathrm{C}($ decomp $)$ 

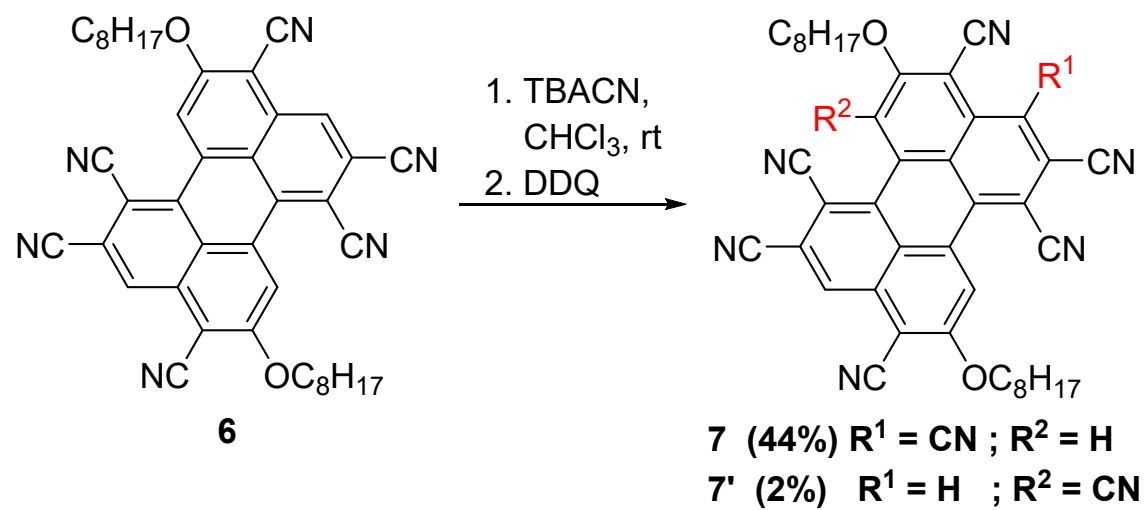

5,11-bis(octyloxy)perylene-1,2,3,4,7,8,10-heptacarbonitrile (7) Under inert atmosphere, compound 6 (45 $\mathrm{mg}, 0.068 \mathrm{mmol})$ was dissolved in $\mathrm{CHCl}_{3}(3.5 \mathrm{~mL})$ and to this solution was slowly added tetrabutylammonium cyanide $\left(0.03 \mathrm{M}\right.$ in $\mathrm{CHCl}_{3}, 2.3 \mathrm{~mL}, 0.068 \mathrm{mmol}$ in $15 \mathrm{~min}$ ) at room temperature. After the completion of the addition, the reaction was stirred at room temperature for another 15 min before 2,3dichloro-5,6-dicyano- $p$-benzoquinone $(31 \mathrm{mg}, 0.137 \mathrm{mmol})$ was added. After another $15 \mathrm{~min}$, the reaction was diluted with $\mathrm{CH}_{2} \mathrm{Cl}_{2}(100 \mathrm{~mL})$ and the solution was washed with water $(50 \mathrm{~mL} \times 3)$. The organic phase was dried over $\mathrm{MgSO}_{4}$ and concentrated. The red brownish crude product was then purified with flash chromatography $\left(\mathrm{SiO}_{2} ; \mathrm{CH}_{2} \mathrm{Cl}_{2}\right)$ to give compound 7 as a red solid (20 mg, $44 \%$ ) and small amount of isomeric 7' as an orange solid ( $1 \mathrm{mg}, 2 \%) \circ$

7: ${ }^{1} \mathrm{H}$ NMR (500 MHz, $\left.\mathrm{CDCl}_{3}\right) \delta(\mathrm{ppm}): 9.18(\mathrm{~s}, 1 \mathrm{H}), 9.11(\mathrm{~s}, 1 \mathrm{H}), 8.72(\mathrm{~s}$, $1 \mathrm{H}), 4.56(\mathrm{t}, J=6.5 \mathrm{~Hz}, 2 \mathrm{H}), 4.52(t, \mathrm{~J}=6.5 \mathrm{~Hz}, 2 \mathrm{H}), 2.06-1.98(\mathrm{~m}, 4 \mathrm{H})$, 
$1.61-1.53(\mathrm{~m}, 4 \mathrm{H}), 1.43-1.23(\mathrm{~m}, 16 \mathrm{H}), 0.88(\mathrm{t}, J=6.5 \mathrm{~Hz}, 6 \mathrm{H}) .{ }^{13} \mathrm{C} \mathrm{NMR}$ $\left(125 \mathrm{MHz}, \mathrm{CDCl}_{3}\right) \delta(\mathrm{ppm}): 166.0,163.4,137.2,134.5,133.6,132.9,132.8$ $132.0,131.0,125.1,124.2,123.0,119.3,118.2,118.1,116.6,116.0,114.4$, $114.2,112.8,112.5,112.1,110.9,107.7,107.5,100.6,98.2,72.4,72.2,31.7$ 29.1, 29.1, 28.9, 28.8, 25.7, 25.6, 22.6, 14.1. HRMS (FAB) m/e: calcd for $\mathrm{C}_{43} \mathrm{H}_{38} \mathrm{O}_{2} \mathrm{~N}_{7}[\mathrm{M}+\mathrm{H}]^{+}:$684.3087 found: 684.3085. IR $v\left(\mathrm{~cm}^{-1}\right): 3056,2926$, $2228(\mathrm{C} \equiv \mathrm{N}$ stretch $), 1635,1595,1422,1366,1147 . \mathrm{UV} / \mathrm{Vis}\left(\mathrm{CH}_{2} \mathrm{Cl}_{2}\right)$ : $\lambda_{\max }=476,510 \mathrm{~nm}$. Fluorescence $\left(\mathrm{CH}_{2} \mathrm{Cl}_{2}, \lambda_{\mathrm{ex}}=510 \mathrm{~nm}\right): \lambda_{\mathrm{em}}=535 \mathrm{~nm} . \Phi=$ 0.26 .

7': ${ }^{1} \mathrm{H}$ NMR (500 MHz, $\left.\mathrm{CDCl}_{3}\right) \delta(\mathrm{ppm}): 9.13$ (s, 1H), $8.80(\mathrm{~s}, 1 \mathrm{H}), 8.73$ (s, 1H), 5.09-5.05 (m, 1H), 4.76-4.71 (m, 1H), 4.57-4.52 (m, 1H), 4.494.45 (m, 1H), 2.05-1.98 (m, 4H), 1.62-1.54 (m, 4H), 1.40-1.23 (m, 16H), 0.89-0.84 (m, 6H). ${ }^{13} \mathrm{C}$ NMR (125 MHz, $\left.\mathrm{CDCl}_{3}\right) \delta(\mathrm{ppm}): 164.6,163.4$, 137.0, 135.1, 134.1, 134.0, 133.7, 131.5, 131.4, 130.5, 124.7, 123.4, 119.4, $118.0,117.4,116.2,115.3,114.2,114.1,113.9,112.8,112.5,111.9,111.7$ 108.7, 100.0, 99.9, 78.0, 71.9, 31.7, 31.7, 29.9, 29.7, 29.1, 29.1, 28.9, 25.7, 25.5, 22.6, 22.6, 14.1. HRMS (EI) m/e: calcd for $\mathrm{C}_{43} \mathrm{H}_{37} \mathrm{O}_{2} \mathrm{~N}_{7}[\mathrm{M}]^{+}$: 683.3009 found: $683.3006 . \mathrm{IR} v\left(\mathrm{~cm}^{-1}\right): 3065,2925,2225(\mathrm{C} \equiv \mathrm{N}$ stretch), 1584, 1457, 1349, 1112. UV/Vis $\left(\mathrm{CH}_{2} \mathrm{Cl}_{2}\right): \lambda_{\max }=468,497 \mathrm{~nm}$. 
Fluorescence $\left(\mathrm{CH}_{2} \mathrm{Cl}_{2}, \lambda_{\mathrm{ex}}=497 \mathrm{~nm}\right): \lambda_{\mathrm{em}}=545 \mathrm{~nm} . \Phi=0.12$. 

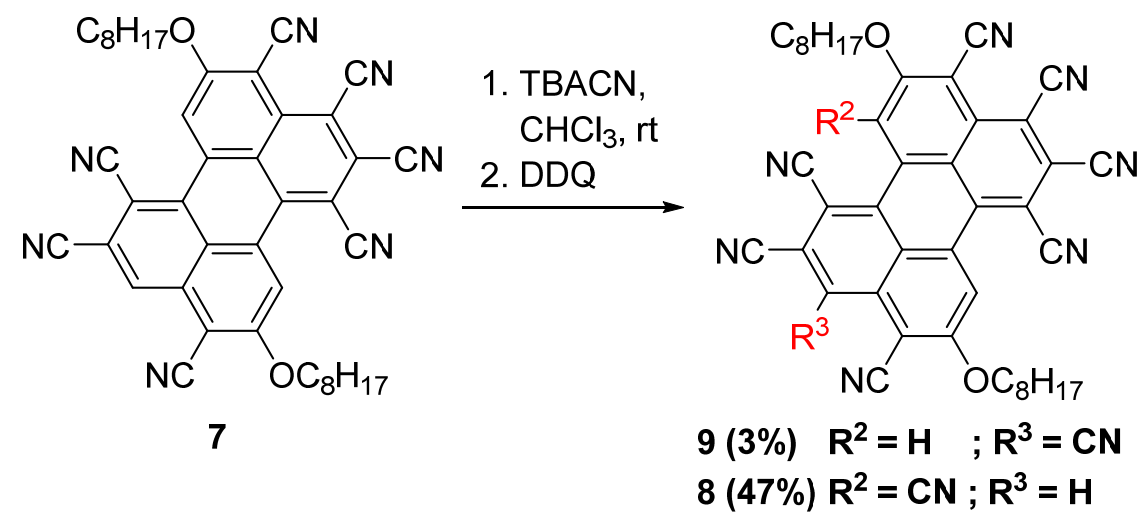

5,11-bis(octyloxy)perylen-1,2,3,4,6,7,8,10-octacarbonitrile (8) and 5,11bis(octyloxy)perylen-1,2,3,4,7,8,9,10-octacarbonitrile (9): Identical procedure was followed as in the preparation of $\mathbf{7}$ and 7 '. Compound 8 (47\%) and 9 (3\%) were obtained as purple red solid.

8: ${ }^{1} \mathrm{H}$ NMR (500 MHz, $\left.\mathrm{CDCl}_{3}\right) \delta(\mathrm{ppm}): 9.14(\mathrm{~s}, 1 \mathrm{H}), 8.77(\mathrm{~s}, 1 \mathrm{H}), 5.12-$ $5.07(\mathrm{~m}, 1 \mathrm{H}), 4.79-4.74(\mathrm{~m}, 1 \mathrm{H}), 4.62-4.57(\mathrm{~m}, 1 \mathrm{H}), 4.53-4.49(\mathrm{~m}, 1 \mathrm{H})$, 2.06-2.01 (m, 4H), 1.60-1.56 (m, 4H), 1.41-1.23 (m, 16H), 0.89-0.85 (m, $6 \mathrm{H}) .{ }^{13} \mathrm{C}$ NMR $\left(125 \mathrm{MHz}, \mathrm{CDCl}_{3}\right) \delta(\mathrm{ppm}): 166.0,164.4,135.8,135.0$, 134.1, 133.3, 132.0, 131.2, 131.1, 124.2, 124.1, 124.1, 119.6, 118.6, 115.9, $115.7,114.4,114.0,113.6,112.7,112.6,112.5,111.9,111.5,110.7,109.4$, 100.7, 98.5, 78.0, 72.5, 31.7, 29.9, 29.7, 29.1, 29.1, 28.8, 25.6, 25.4, 22.6, 14.1. HRMS (FAB) m/e: calcd for $\mathrm{C}_{44} \mathrm{H}_{37} \mathrm{O}_{2} \mathrm{~N}_{8}[\mathrm{M}+\mathrm{H}]^{+}: 709.3039$ found: 709.3033. IR $v\left(\mathrm{~cm}^{-1}\right): 2926,2228(\mathrm{C} \equiv \mathrm{N}$ stretch), 1587, 1426, 1354, 1143. UV/Vis $\left(\mathrm{CH}_{2} \mathrm{Cl}_{2}\right): \lambda_{\max }=488,517 \mathrm{~nm}$. Fluorescence $\left(\mathrm{CH}_{2} \mathrm{Cl}_{2}, \lambda_{\mathrm{ex}}=517 \mathrm{~nm}\right)$ : $\lambda_{\mathrm{em}}=565 \mathrm{~nm} . \Phi=0.08$. 
9: ${ }^{1} \mathrm{H}$ NMR (500 MHz, acetone-d 6 ) $\delta$ (ppm): 9.46 (br, 1H), 4,72 (t, $J=6.5$ $\mathrm{Hz}, 4 \mathrm{H}$ ), 1.63 (quin, $J=7.5 \mathrm{~Hz}, 4 \mathrm{H}), 1.48-1.29(\mathrm{~m}, 20 \mathrm{H}), 0.89$ (t, $J=6.5$ $\mathrm{Hz}, 6 \mathrm{H}) .{ }^{13} \mathrm{C}$ NMR $(125 \mathrm{MHz}$, acetone-d 6 ) $\delta$ (ppm): 166.8, 138.1, 133.4, $132.6,125.9,125.2,121.5,117.4,115.3,114.8,113.9,112.4,110.2,99.4$ $72.8,32.6,30.5,30.3,30.0,26.5,23.4,14.4$. HRMS (FAB) m/e: calcd for $\mathrm{C}_{44} \mathrm{H}_{37} \mathrm{O}_{2} \mathrm{~N}_{8}[\mathrm{M}+\mathrm{H}]^{+}: 709.3039$ found: 709.3036. IR $v\left(\mathrm{~cm}^{-1}\right): 2923,2229$ (C $\equiv \mathrm{N}$ stretch), 1597, 1425, 1366, 1142. UV/Vis $\left(\mathrm{CH}_{2} \mathrm{Cl}_{2}\right): \lambda_{\max }=487,522$ nm. Fluorescence $\left(\mathrm{CH}_{2} \mathrm{Cl}_{2}, \lambda_{\mathrm{ex}}=522 \mathrm{~nm}\right): \lambda_{\mathrm{em}}=545 \mathrm{~nm} . \Phi=0.30$. 


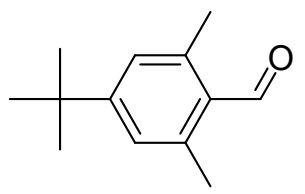

The synthesis of 4-tert-butyl-2,6-dimethylbenzaldehyde was carried out according to the literature. J. Am. Chem. Soc., 2015, 137 (1), 142-145. The ${ }^{1} \mathrm{H}$ NMR, ${ }^{13} \mathrm{C}$ NMR spectrum are identical with the reported values.

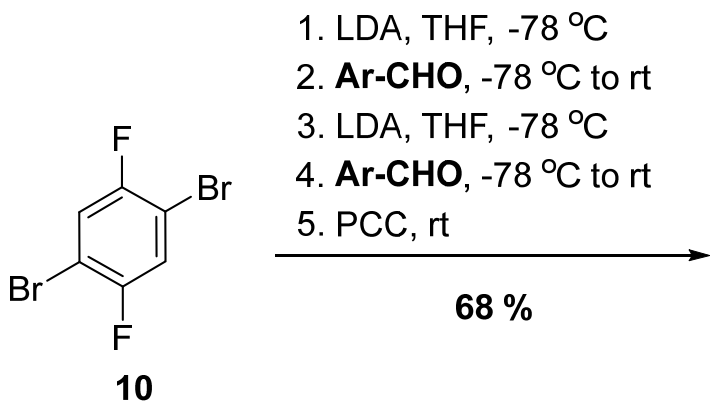<smiles>O=C([Al])c1c(F)c(Br)c(C(=O)[Al])c(F)c1Br</smiles>

11

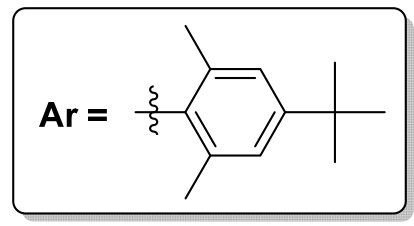

(2,5-dibromo-3,6-difluoro-1,4-phenylene)bis((4-(tert-butyl)-2,6-

dimethylphenyl)methanone) (11)

In a glove box, compound 10 (2,5-difluoro 1,4-dibromobenzene, $500 \mathrm{mg}$, $1.839 \mathrm{mmol})$ was dissolved in THF $(60 \mathrm{~mL})$. The solution was cooled to $-78{ }^{\circ} \mathrm{C}$ for $30 \mathrm{~min}$. Lithium diisopropylamide solution $(1.0 \mathrm{M}$ in THF/hexanes, $2.8 \mathrm{~mL}, 2.758 \mathrm{mmol}$ ) was slowly added to the chilled solution and the lithiation proceeded at low temperature for $30 \mathrm{~min}$. A THF solution of 4-tert-butyl-2,6-dimethylbenzaldehyde (525 mg, 2.758 mmol in $10 \mathrm{~mL}$ THF) was slowly added to the reaction before it was warmed back to room temperature and stirred for $30 \mathrm{~min}$. The reaction 
was cooled back to $-78{ }^{\circ} \mathrm{C}$. The previous steps (LDA, then 4-tert-butyl2,6-dimethylbenzaldehyde) were repeated to install the second benzoyl moiety. The reaction was warmed back to room temperature again and stirred for another $30 \mathrm{~min}$. Pyridinium chlorochromate $(2.0 \mathrm{~g}, 9.195$ mmol) was added and the reaction was stirred at room temperature for another $24 \mathrm{hr}$. To the reaction was added aqueous $\mathrm{HCl}(1 \mathrm{M}, 200 \mathrm{~mL})$ and the mixture was stirred overnight. The reaction was extracted with $\mathrm{CH}_{2} \mathrm{Cl}_{2}$ (100 mL x 3). The combined organic phase was dried over $\mathrm{MgSO}_{4}$ and concentrated to give the crude product. After the crude mixture was washed with EtOH (100 mL), pure compound 11 was obtained as a white solid ( $811 \mathrm{mg}, 68 \%)$.

${ }^{1} \mathrm{H}$ NMR (400 MHz, $\left.\mathrm{CDCl}_{3}\right) \delta(\mathrm{ppm}): 7.04(\mathrm{~s}, 4 \mathrm{H}), 2.25$ (s, 12H), 1.29 (s, 18H). $\left.{ }^{13} \mathrm{C} \mathrm{NMR} \mathrm{(100} \mathrm{MHz,} \mathrm{CDCl}_{3}\right) \delta(\mathrm{ppm}): 192.6,154.5,153.3(\mathrm{~d}, J=$ $251 \mathrm{~Hz}), 136.7,135.5,133.1(\mathrm{~d}, J=20 \mathrm{~Hz}), 126.2,108.7(\mathrm{~d}, J=29 \mathrm{~Hz})$, 34.6, 31.0, 21.0. HRMS (EI) $\mathrm{m} / \mathrm{e}$ : calcd for $\mathrm{C}_{32} \mathrm{H}_{34} \mathrm{O}_{2} \mathrm{Br}_{2} \mathrm{~F}_{2}[\mathrm{M}]^{+}$: 646.0894 found: $646.0886 . \quad \operatorname{IR} v\left(\mathrm{~cm}^{-1}\right): 2962,1680,1410,1240,970,870$, 833. 
<smiles>O=C([Al])c1c(F)c(Br)c(C(=O)[Al])c(Br)c1F</smiles>

11

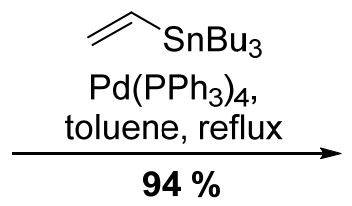<smiles>C=Cc1c(F)c(C=C)c(C(=O)Br)c(F)c1C(=O)Br</smiles>

12

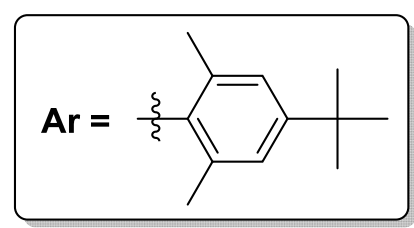

(2,5-difluoro-3,6-divinyl-1,4-phenylene)bis((4-(tert-butyl)-2,6-

dimethylphenyl)methanone) (12) Under inert atmosphere, compound 11 (3.5 g, $5.398 \mathrm{mmol})$ and tetrakis(triphenylphosphine)palladium(0) (312 mg, $0.270 \mathrm{mmol})$ was dissolved in toluene $(60 \mathrm{~mL})$. To this solution was then added tri- $n$-butyl vinyltin $(4.0 \mathrm{~mL}, 4.03 \mathrm{~g}, 13.490 \mathrm{mmol})$. The reaction was refluxed for $8 \mathrm{hr}$ before cooled to room temperature and diluted with $\mathrm{CH}_{2} \mathrm{Cl}_{2}(200 \mathrm{~mL})$. The solution was passed through a celite pad and concentrated. The brownish crude product was washed with EtOH (300 $\mathrm{mL})$ to give compound $\mathbf{1 2}$ as a white solid (2.75 g, $94 \%)$.

${ }^{1} \mathrm{H}$ NMR (400 MHz, $\left.\mathrm{CDCl}_{3}\right) \delta(\mathrm{ppm}): 7.02(\mathrm{~s}, 4 \mathrm{H}), 6.52(\mathrm{dd}, J=18.0,12.0$ $\mathrm{Hz}, 2 \mathrm{H}), 5.78(\mathrm{~d}, J=18.0 \mathrm{~Hz}, 2 \mathrm{H}), 5.53(\mathrm{~d}, J=12.0 \mathrm{~Hz}, 2 \mathrm{H}), 2.22(\mathrm{~s}, 12 \mathrm{H})$, $1.29(\mathrm{~s}, 18 \mathrm{H}) . \quad{ }^{13} \mathrm{C}$ NMR $\left(100 \mathrm{MHz} \mathrm{CDCl}_{3}\right) \delta(\mathrm{ppm}): 196.5,154.4(\mathrm{~d}, J$ $=252 \mathrm{~Hz}), 153.7,137.2,136.1,131.9(\mathrm{~d}, J=20 \mathrm{~Hz}), 126.7,126.0,124.9$ $(\mathrm{d}, J=19 \mathrm{~Hz}), 124.1,34.6,31.1,20.9$. HRMS (EI) m/e: calcd for 
$\mathrm{C}_{36} \mathrm{H}_{40} \mathrm{O}_{2} \mathrm{~F}_{2}[\mathrm{M}]^{+}: 542.2996$ found: 542.2991. IR $v\left(\mathrm{~cm}^{-1}\right): 2964,1664$, 1600, 1446, 1427, 1294, 1251, 1126, 970, 937, 889, 865, 779, 657.<smiles>C=Cc1c(F)c(C(=O)Br)c(C=C)c(F)c1C(=O)Br</smiles>

12
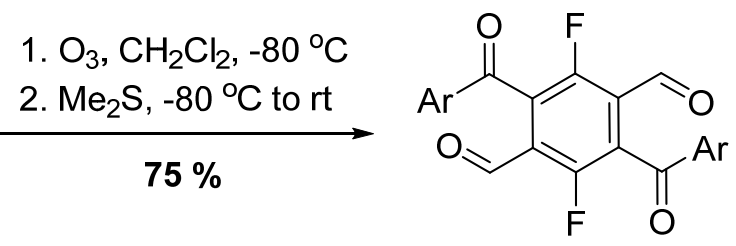

13

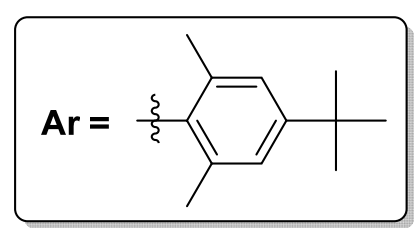

2,5-bis(4-(tert-butyl)-2,6-dimethylbenzoyl)-3,6-

difluoroterephthalaldehyde (13): Compound 12 (300 mg, $0.522 \mathrm{mmol}$ ) was dissolved in $\mathrm{CH}_{2} \mathrm{Cl}_{2}(200 \mathrm{~mL})$. The solution was cooled to $-80{ }^{\circ} \mathrm{C}$ and ozone was bubbled to the reaction for $5 \mathrm{~min}$. The completion of the ozonolysis was indicated by the solution's lightly green color and TLC. Dimethyl sulfide $(0.203 \mathrm{~mL}, 2.764 \mathrm{mmol})$ was added to the reaction before it was warmed back to room temperature. The reaction was washed with water $(100 \mathrm{~mL} \times 3)$. The organic phase was dried over $\mathrm{MgSO}_{4}$ and concentrated. The crude grey solid was washed with $i-\mathrm{PrOH}(100 \mathrm{~mL})$ to give purified 13 as a white solid (226 mg, $75 \%$ ).

${ }^{1} \mathrm{H}$ NMR (400 MHz, $\left.\mathrm{CDCl}_{3}\right) \delta(\mathrm{ppm}): 10.15$ (s, 2H), 7.06 (s, 4H), 2.28 (s, 12H), $1.29(\mathrm{~s}, 18 \mathrm{H}) . \quad{ }^{13} \mathrm{C} \mathrm{NMR}\left(100 \mathrm{MHz}, \mathrm{CDCl}_{3}\right) \delta(\mathrm{ppm}): 192.8,185.5$, $155.7(\mathrm{~d}, J=265 \mathrm{~Hz}), 154.6,136.4,135.2,134.0(\mathrm{~d}, J=18 \mathrm{~Hz}), 129.0(\mathrm{~d}$, $J=16 \mathrm{~Hz}), 126.3,34.6,31.0,21.01$. HRMS (EI) m/e: calcd for 
$\mathrm{C}_{34} \mathrm{H}_{36} \mathrm{O}_{4} \mathrm{~F}_{2}[\mathrm{M}]^{+}: 546.2582$ found: $546.2584 . \quad \mathrm{IR} v\left(\mathrm{~cm}^{-1}\right):$ 2956, 2906, $2867,1710,1662,1602,1429,1396,1294,1274,1230,1124,941,867$, $827,678$.<smiles>O=Cc1c(F)c(C(=O)Br)c(C(=O)Br)c(F)c1C=O</smiles>

13

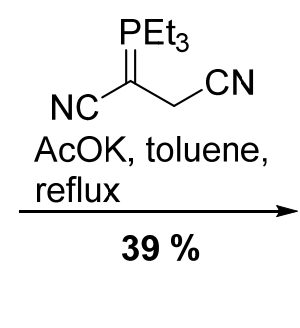<smiles>N#Cc1cc2c(F)c3c(Br)c(C#N)c(C#N)c(Br)c3c(F)c2cc1C#N</smiles>

14

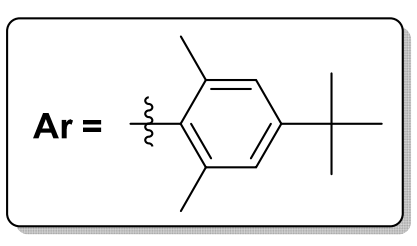

1,5-bis(4-(tert-butyl)-2,6-dimethylphenyl)-9,10-difluoroanthracene-

2,3,6,7-tetracarbonitrile (14)

The required Wittig reagent was prepared to the following procedure :

Under inert atmosphere, fumaronitrile ( $43 \mathrm{mg}, 0.551 \mathrm{mmol}$ ) was dissolved in THF $(5 \mathrm{~mL})$ and to this solution was added triethylphosphine $(1.0 \mathrm{M}$ in THF, $0.55 \mathrm{~mL}, 0.55 \mathrm{mmol}$ ). The mixture was refluxed overnight.

In another flask, compound $13(100 \mathrm{mg}, 0.183 \mathrm{mmol})$ and potassium acetate $(180 \mathrm{mg}, 1.829 \mathrm{mmol})$ was suspended in toluene $(20 \mathrm{~mL})$. The Wittig reagent previously generated $(0.55$ mmole, 3 eq) was slowly added to this toluene solution and the reaction was refluxed for $24 \mathrm{hr}$. The volatiles were then removed in vacuo and the residue was dissolved in $\mathrm{CH}_{2} \mathrm{Cl}_{2}(50 \mathrm{~mL})$. The solution was washed with water $(50 \mathrm{~mL} \times 3)$. The organic phase was dried over $\mathrm{MgSO}_{4}$ and concentrated. The brown crude 
product was purified with flash chromatography $\left(\mathrm{SiO}_{2} ; \mathrm{CH}_{2} \mathrm{Cl}_{2}\right.$ /hexane, 50 \%) to give the anthracene product $\mathbf{1 4}$ as a bright yellow solid (45 $\mathrm{mg}, 39$ $\%)$.

${ }^{1} \mathrm{H}$ NMR (500 MHz, $\left.\mathrm{CDCl}_{3}\right) \delta(\mathrm{ppm}): 8.66(\mathrm{~s}, 2 \mathrm{H}), 7.23(\mathrm{~s}, 4 \mathrm{H}), 1.97$ (s, 12H), $1.39(\mathrm{~s}, 18 \mathrm{H}) .{ }^{13} \mathrm{C} \mathrm{NMR}\left(125 \mathrm{MHz}, \mathrm{CDCl}_{3}\right) \delta(\mathrm{ppm}): 152.8,151.7$ $(\mathrm{d}, J=265 \mathrm{~Hz}), 147.1,134.1,132.1,129.3,125.2,121.6(\mathrm{~m}), 119.4(\mathrm{~m})$, 114.9, 114.1, 113.6, 112.6, 34.6, 31.3, 20.5. HRMS (EI) m/e: calcd for $\mathrm{C}_{42} \mathrm{H}_{36} \mathrm{~N}_{4} \mathrm{~F}_{2}[\mathrm{M}]^{+}: 634.2908$ found: 634.2903. IR $v\left(\mathrm{~cm}^{-1}\right): 2966,2227(\mathrm{C}$ $\equiv \mathrm{N}$ stretch), 1602, 1429, 1371, 1761, 1222, 894, 879, 765, 750.<smiles>N#Cc1cc2c(F)c3c(Br)c(C#N)c(C#N)cc3c(F)c2c(Br)c1C#N</smiles><smiles>[R]c1c(C#N)c(C#N)c([Al])c2c(N)c3cc(C#N)c(C#N)c([Al])c3c(C#N)c12</smiles>

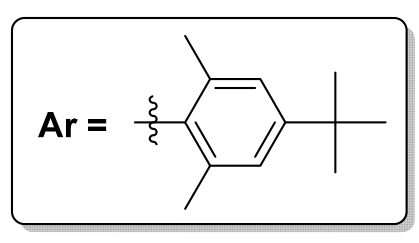

$15 \mathrm{R}=\mathrm{H} \quad(22 \%)$

$16 \mathrm{R}=\mathrm{CN} \quad(10 \%)$

1,5-bis(4-(tert-butyl)-2,6-dimethylphenyl)anthracene-2,3,6,7,9,10-

hexacarbonitrile (15)

Under inert atmosphere, compound $14(180 \mathrm{mg}, 0.283 \mathrm{mmol})$ was dissolved in THF $(14 \mathrm{~mL})$. A THF solution of tetrabutylammonium cyanide (0.3 M, $2 \mathrm{~mL}, 0.6 \mathrm{mmol})$ was slowly added to the solution of $\mathbf{1 4}$ at room temperature. After $15 \mathrm{~min}$, the reaction was diluted with $\mathrm{CH}_{2} \mathrm{Cl}_{2}$ 
$(100 \mathrm{~mL})$ and the mixture was washed with water $(100 \mathrm{~mL} \mathrm{x} \mathrm{3).} \mathrm{The}$ organic phase was dried over $\mathrm{MgSO}_{4}$ and concentrated. The red brown crude product was purified by flash chromatography $\left(\mathrm{SiO}_{2} ; \mathrm{CH}_{2} \mathrm{Cl}_{2} /\right.$ hexane, $70 \%)$ to give compound $\mathbf{1 5}(40 \mathrm{mg}, 22 \%)$ and $\mathbf{1 6}(19 \mathrm{mg}, 10 \%)$ as orange solids.

${ }^{1} \mathrm{H}$ NMR (500 MHz, $\left.\mathrm{CDCl}_{3}\right) \delta(\mathrm{ppm}): 9.08$ (s, 2H), 7.30 (s, 4H), 1.93 (s, 12H), $1.41(\mathrm{~s}, 18 \mathrm{H}) .{ }^{13} \mathrm{C} \mathrm{NMR}\left(125 \mathrm{MHz}, \mathrm{CDCl}_{3}\right) \delta(\mathrm{ppm}): 156.1,149.34$, 136.39, 135.16, 134.18, 131.93, 128.99, 126.04, 116.59, 115.94, 115.46, 114.19, 113.15, 111.5, 34.89, 31.22, 20.58. HRMS (EI) m/e: calcd for $\mathrm{C}_{44} \mathrm{H}_{36} \mathrm{~N}_{6}[\mathrm{M}]^{+}: 648.3001$ found: 648.3010. IR $v\left(\mathrm{~cm}^{-1}\right): 2967,2227(\mathrm{C} \equiv$ N stretch), 1606, 1444, 1363, 1274, 1259, 897, 877, 763, 750.<smiles>N#Cc1cc2c(C#N)c3c(Br)c(C#N)c(C#N)cc3c(C#N)c2c([Al])c1C#N</smiles>

15

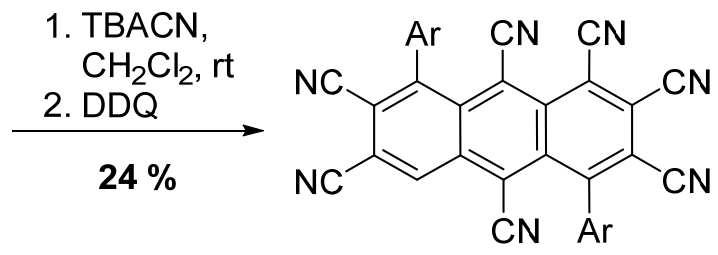

16

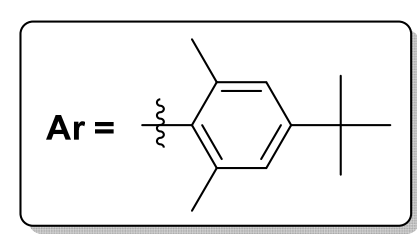$$
\text { , }
$$ 


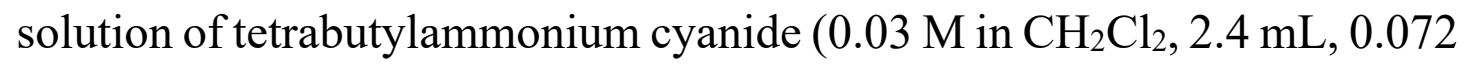
mmol) over $15 \mathrm{~min}$. After the reaction was stirred for another $15 \mathrm{~min}$ at room temperature, 2,3-dichloro-5,6-dicyano- $p$-benzoquinone (17 mg, $0.074 \mathrm{mmol}$ ) was added. The reaction was allowed to proceed for another 15 min before diluted with $\mathrm{CH}_{2} \mathrm{Cl}_{2}(100 \mathrm{~mL})$ and washed with water (50 $\mathrm{mL} \times 3$ ). The organic phase was dried over $\mathrm{MgSO}_{4}$ and concentrated. The red brown crude product was purified by flash chromatography $\left(\mathrm{SiO}_{2}\right.$; $\left.\mathrm{CH}_{2} \mathrm{Cl}_{2}\right)$ to give product $\mathbf{1 6}(10 \mathrm{mg}, 24 \%)$ as an orange solid. Notable amount of starting material 15 was also recovered (10 mg, $25 \%$ ).

${ }^{1} \mathrm{H}$ NMR (500 MHz, $\left.\mathrm{CDCl}_{3}\right) \delta(\mathrm{ppm}): 9.08$ (s, 2H), 7.32 (s, 2H), 7.30 (s, 2H), $1.95(\mathrm{~s}, 6 \mathrm{H}), 1.94(\mathrm{~s}, 6 \mathrm{H}), 1.41(\mathrm{~s}, 9 \mathrm{H}), 1.39(\mathrm{~s}, 9 \mathrm{H}) .{ }^{13} \mathrm{C}$ NMR (125 $\left.\mathrm{MHz}, \mathrm{CDCl}_{3}\right) \delta(\mathrm{ppm}): 157.2,156.0,153.2,149.8,136.2,135.9,135.5$, $134.0,133.8,132.2,132.2,129.2,128.8,126.4,126.3,124.7,118.4,117.2$ $116.9,116.8,116.6,114.3,113.9,113.0,112.6,112.1,111.7,111.1,110.0$ 35.0, 34.9, 31.2, 31.2, 20.7, 20.6. HRMS (ESI) m/e: calcd for $\mathrm{C}_{45} \mathrm{H}_{35} \mathrm{~N}_{7}$ [M] : 673.2954 found: $673.2948 . \quad$ IR $v\left(\mathrm{~cm}^{-1}\right): 2952,2921,2852,2239(\mathrm{C}$ $\equiv \mathrm{N}$ stretch), 1608, 1457, 1377, 1363, 1275, 1259, 867, 750. 


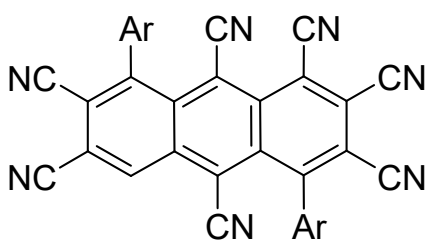

16
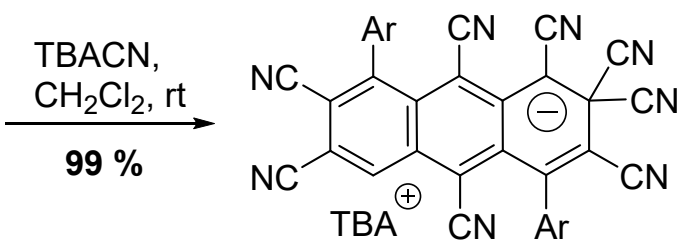

17

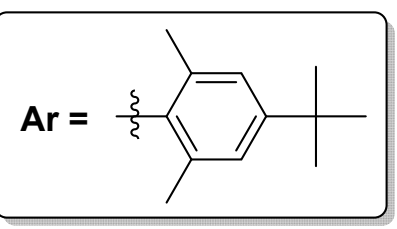

(Salt 17)

Under inert atmosphere, compound 16 (5 mg, $0.007 \mathrm{mmol})$ was dissolved in $\mathrm{CH}_{2} \mathrm{Cl}_{2} \quad(2 \mathrm{~mL})$. To this solution was slowly added tetrabutylammonium cyanide $\left(0.03 \mathrm{M}\right.$ in $\left.\mathrm{CH}_{2} \mathrm{Cl}_{2}, 0.26 \mathrm{~mL}, 0.008 \mathrm{mmol}\right)$. After stirred for $15 \mathrm{~min}$ at room temperature, the reaction mixture was diluted with $\mathrm{CH}_{2} \mathrm{Cl}_{2}(50 \mathrm{~mL})$ and washed with water $(20 \mathrm{~mL} \times 3)$. The organic phase was dried over $\mathrm{MgSO}_{4}$ and concentrated. The red crude product was purified by flash chromatography $\left(\mathrm{SiO}_{2} ; \mathrm{EtOAc} / \mathrm{CH}_{2} \mathrm{Cl}_{2}=5\right.$ \%) to furnish anion 17 as a red solid (7 mg, $99 \%$ ).

${ }^{1} \mathrm{H}$ NMR (500 MHz, $\left.\mathrm{CDCl}_{3}\right) \delta(\mathrm{ppm}): 7.92$ (s, 1H), 7.17 (s, 2H), $7.11(\mathrm{~s}$, 2H), $3.08(\mathrm{t}, J=7.5 \mathrm{~Hz}, 8 \mathrm{H}), 2.05(\mathrm{~s}, 6 \mathrm{H}), 1.97(\mathrm{~s}, 6 \mathrm{H}), 1.56(\mathrm{br}, 8 \mathrm{H}), 1.42-$ $1.37(\mathrm{~m}, 8 \mathrm{H}), 1.35(\mathrm{~s}, 9 \mathrm{H}), 1.33(\mathrm{~s}, 9 \mathrm{H}), 0.98(\mathrm{t}, J=7.0 \mathrm{~Hz}, 12 \mathrm{H})$.

${ }^{13} \mathrm{C}$ NMR $\left(125 \mathrm{MHz}, \mathrm{CDCl}_{3}\right) \delta(\mathrm{ppm}): 154.7,152.3,146.2,141.6,139.8$, $138.7,136.2,136.1,131.3,129.7,128.7,125.2,124.7,122.9,119.4,118.4$, $117.9,116.7,116.1,114.6,114.6,114.3,114.2,114.2,111.4,107.2,106.4$ 104.9, 74.9, 59.1, 34.7, 34.5, 31.4, 31.3, 23.9, 20.4, 20.3, 19.7, 13.6. HRMS 
(ESI) m/e: calcd for $\mathrm{C}_{46} \mathrm{H}_{35} \mathrm{~N}_{8}{ }^{-}$[octanitrile anion without the cationic TBA]:

699.2985 found: 699.2976. IR $v\left(\mathrm{~cm}^{-1}\right): 2960,2923,2217(\mathrm{C} \equiv \mathrm{N}$ stretch), $2179(\mathrm{C} \equiv \mathrm{N}$ stretch $), 1542,1417,1388,1242,869,736$. 


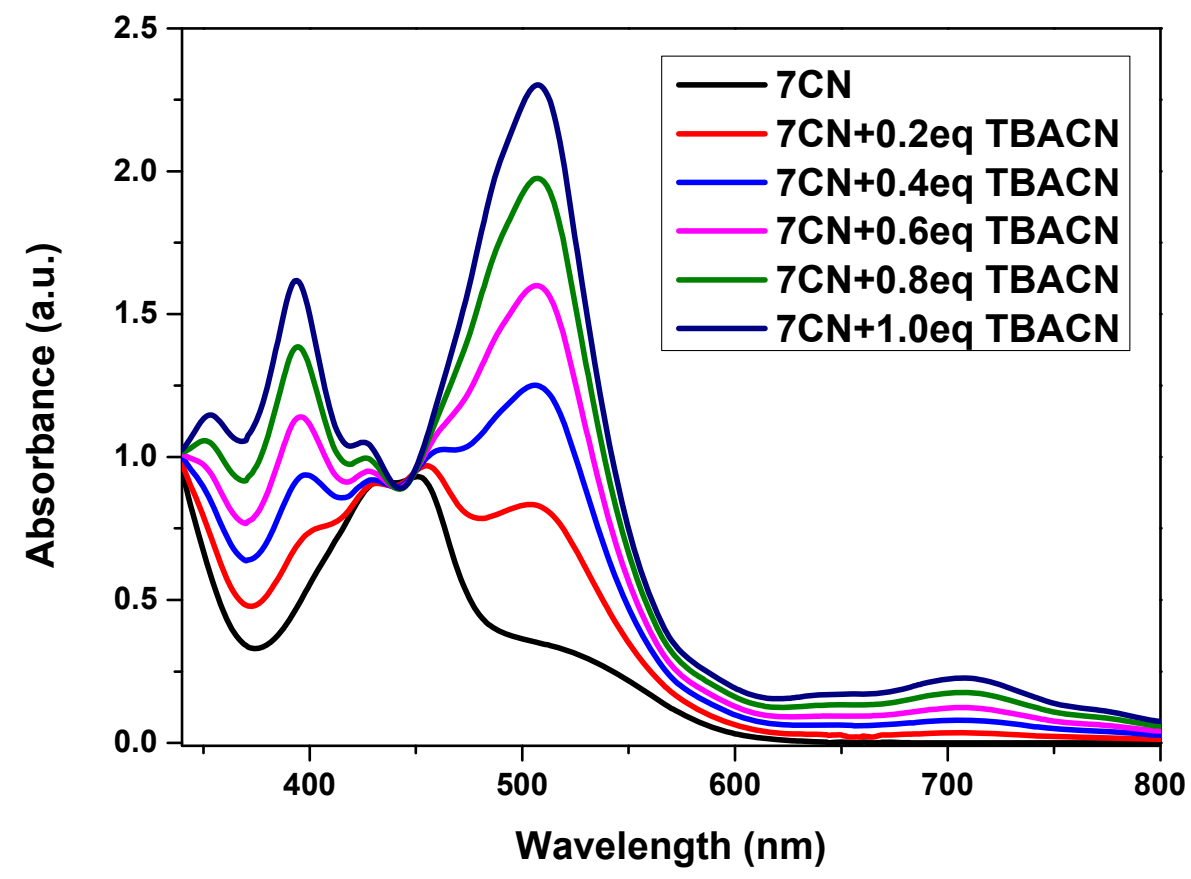

Figure 1 (SI1): UV-vis titration of 16 with TBACN $\left(\mathrm{CH}_{2} \mathrm{Cl}_{2}\right)$

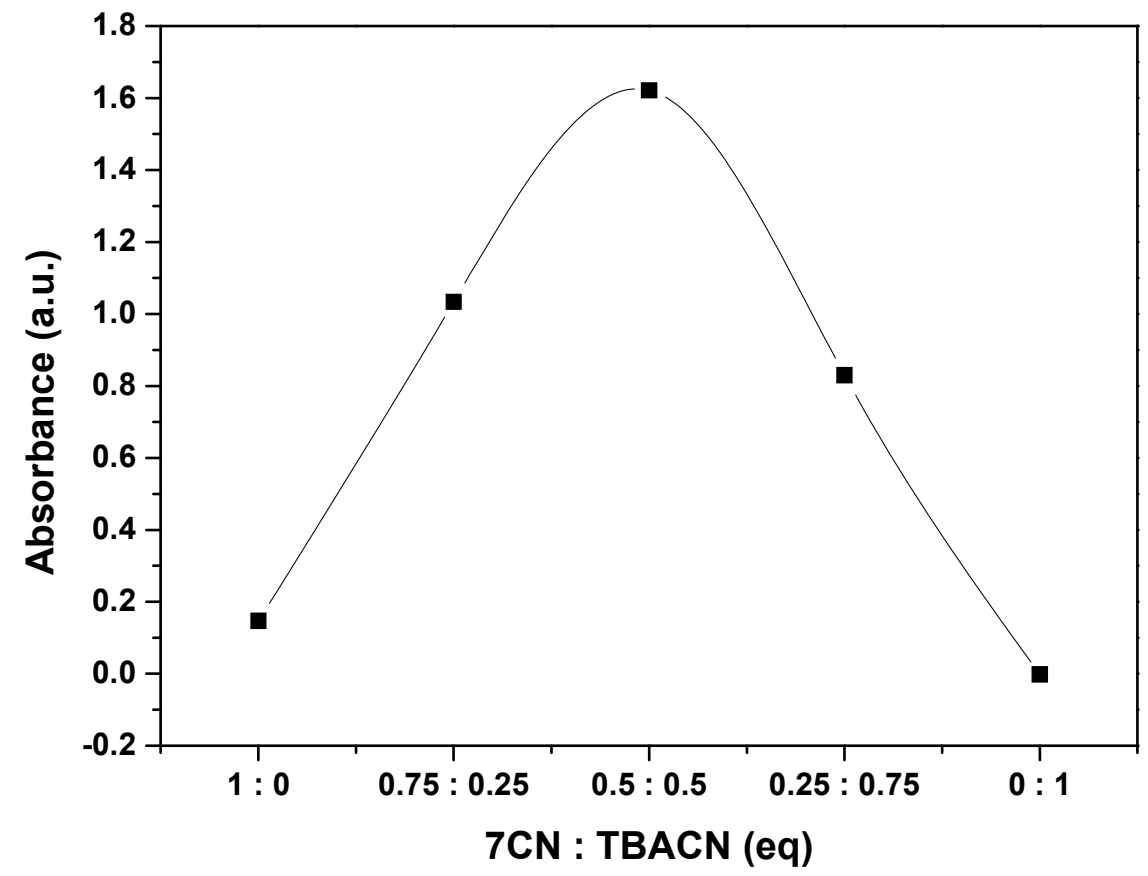

Figure 2 (SI1): Job plot for $16+\mathrm{TBACN}\left(\mathrm{CH}_{2} \mathrm{Cl}_{2}\right)$ 


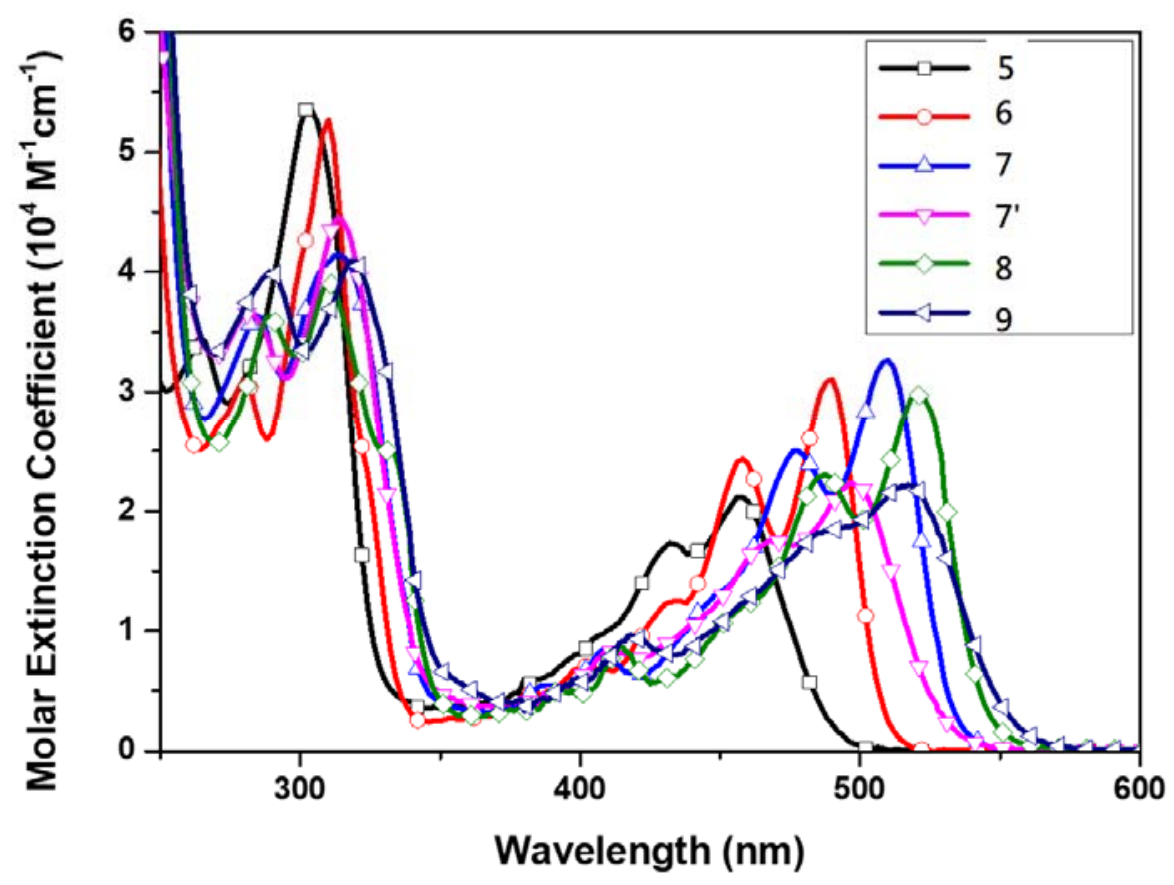

Figure 3 (SI1): UV-vis spectrum for 5-9 $\left(\mathrm{CH}_{2} \mathrm{Cl}_{2}\right)$

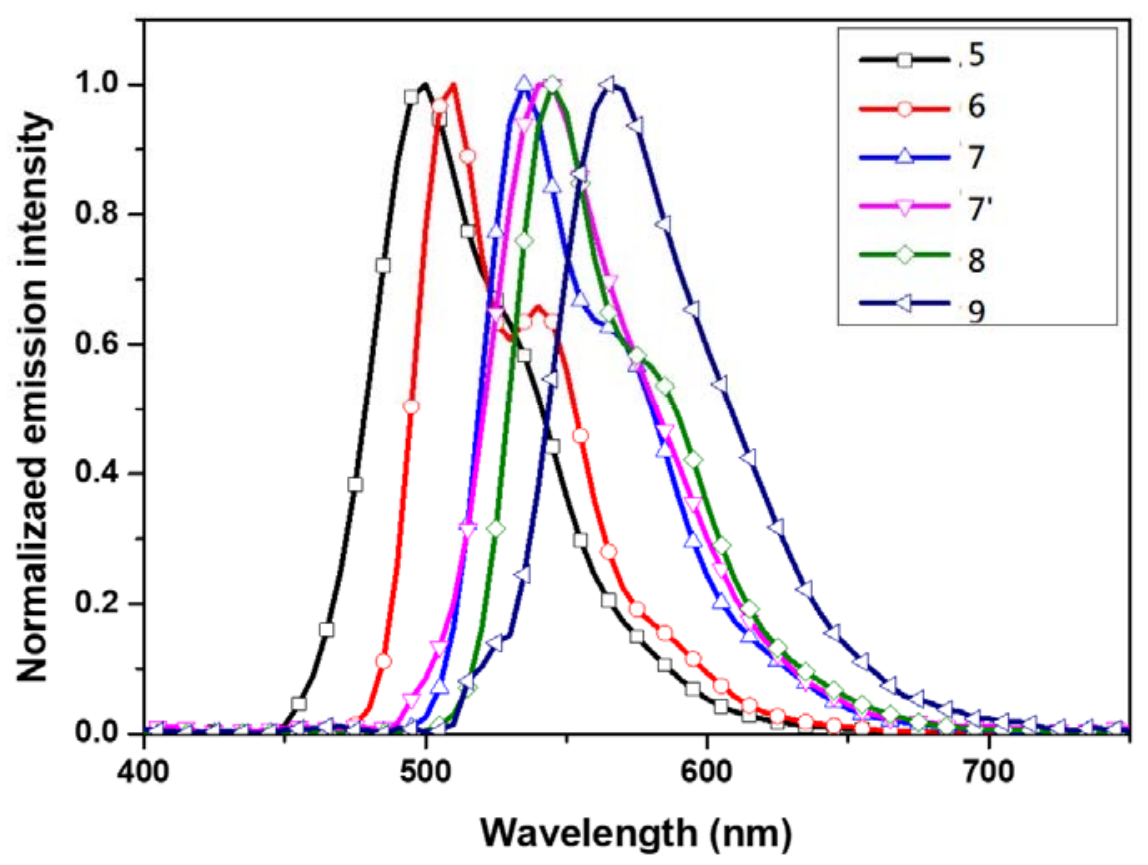

Figure 4 (SI1): Normalized fluorescence spectrum for $\mathbf{5 - 9}\left(\mathrm{CH}_{2} \mathrm{Cl}_{2}\right)$ 


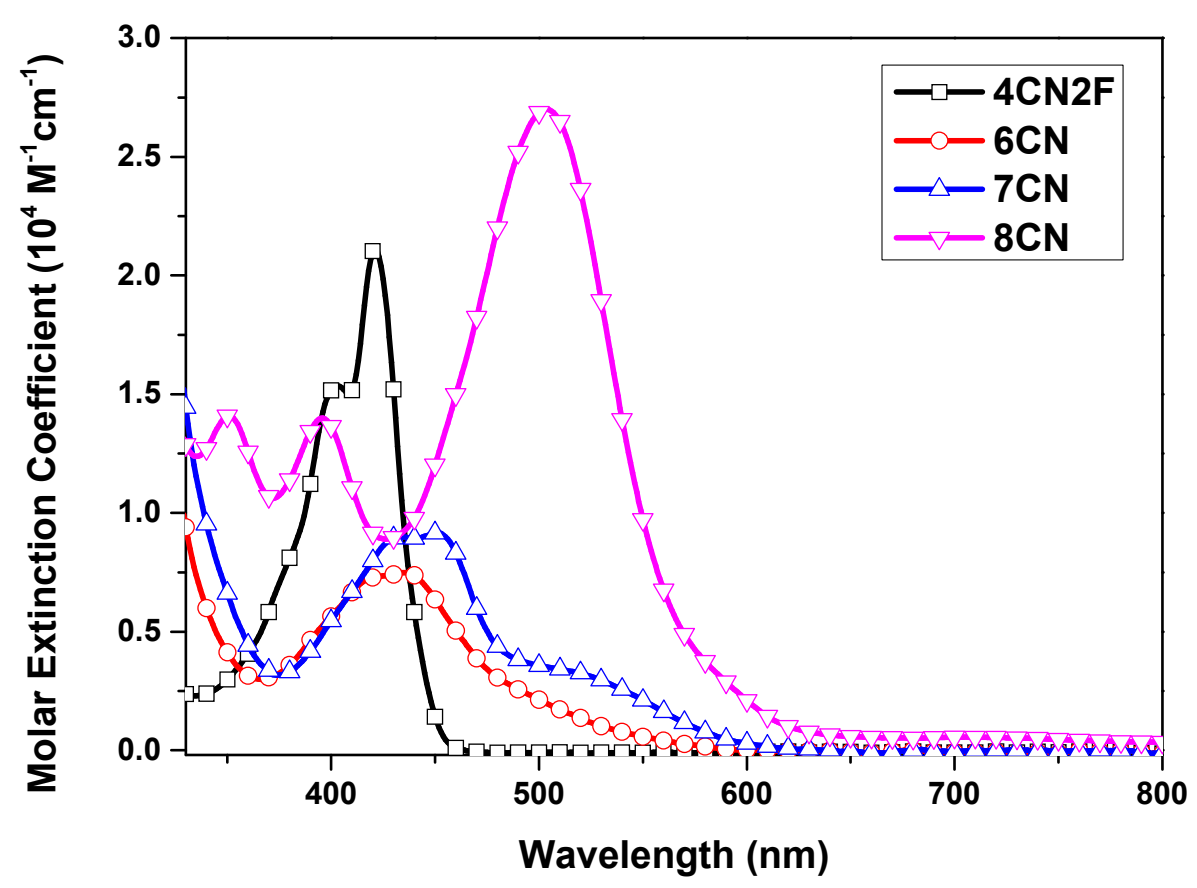

Figure 5 (SI1): UV-vis spectrum for 14-17 $\left(\mathrm{CH}_{2} \mathrm{Cl}_{2}\right)$

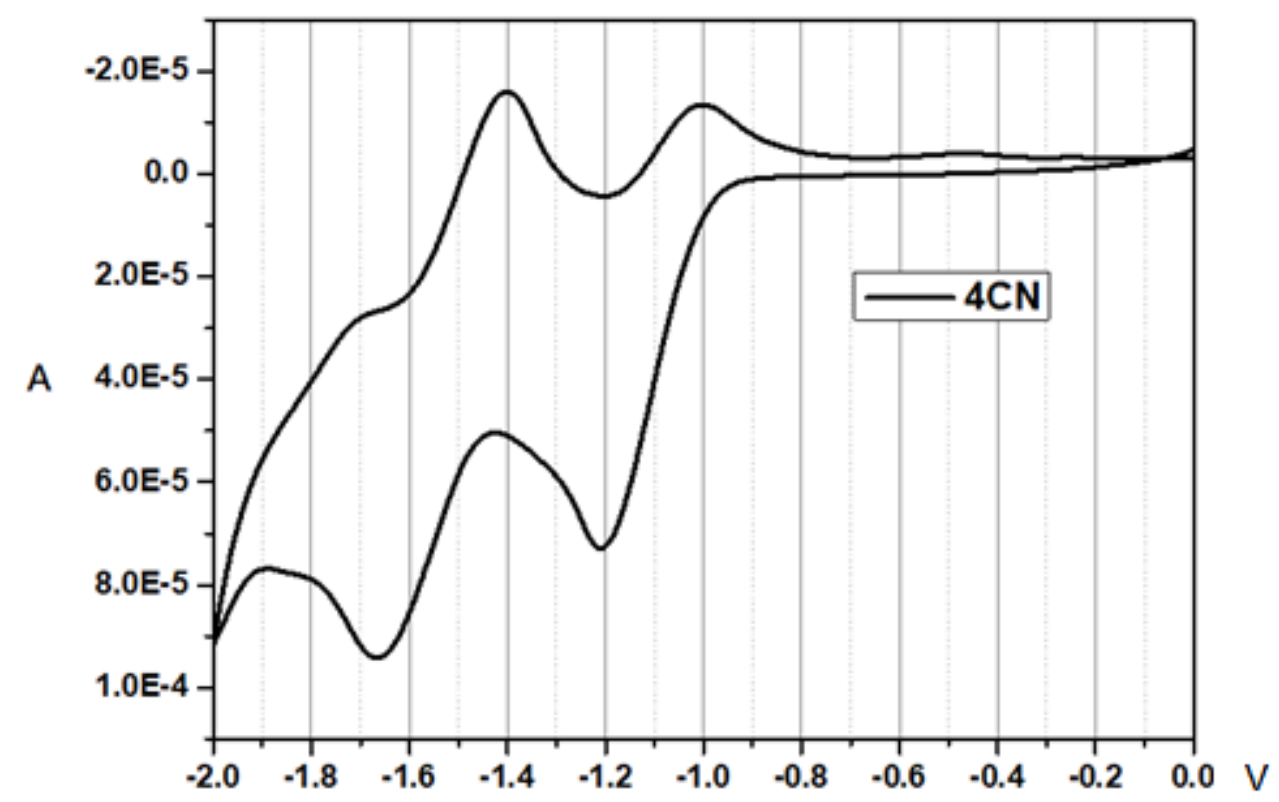

Figure 5 (SI1): Cyclic voltammetry for 5 


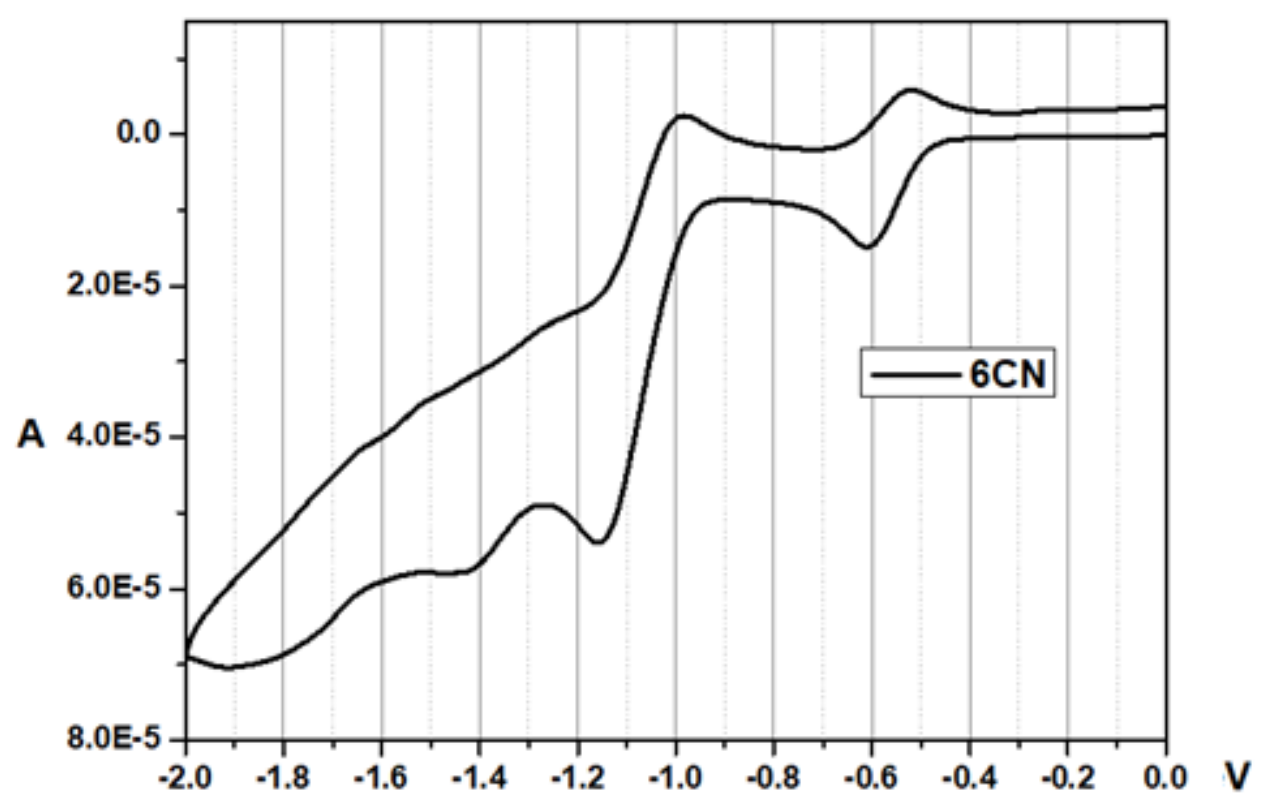

Figure 6 (SI1): Cyclic voltammetry for 6

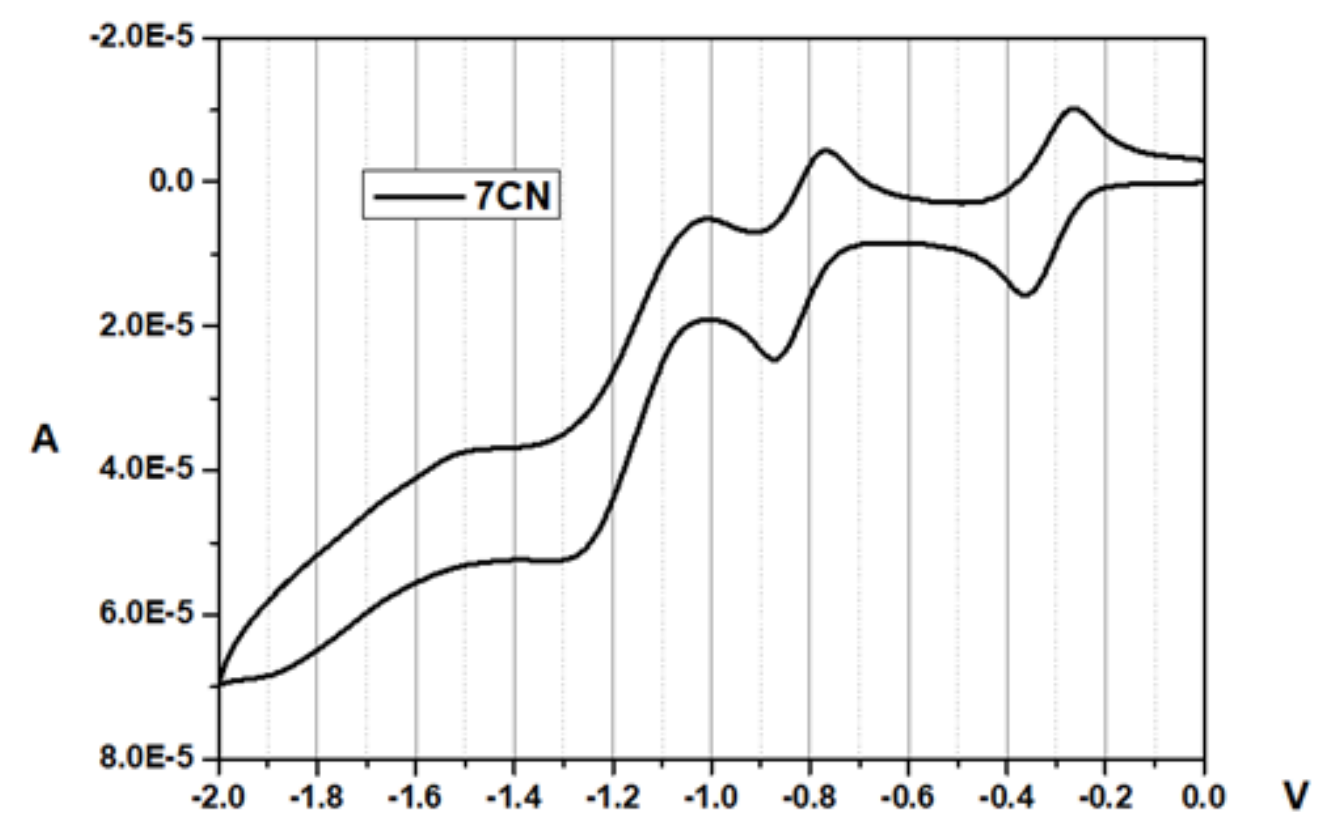

Figure 7 (SI1): Cyclic voltammetry for 7 


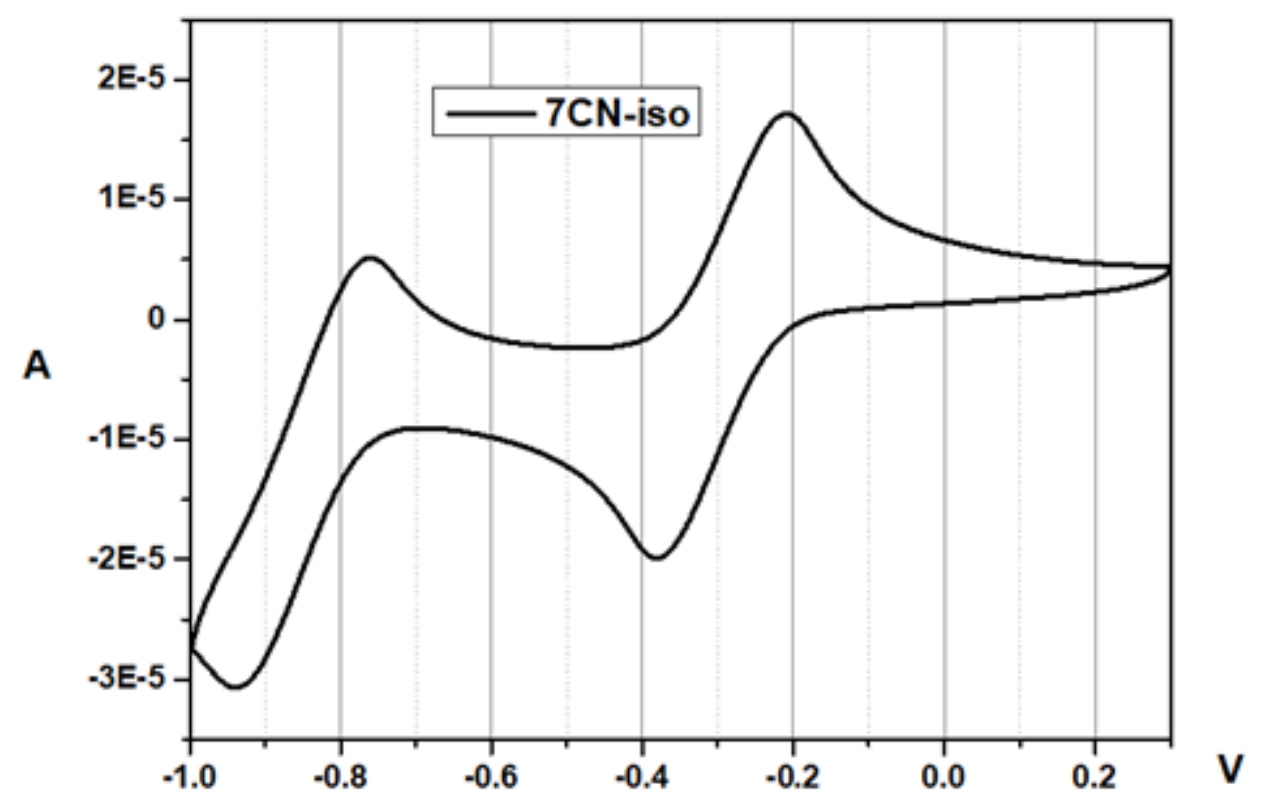

Figure 8 (SI1): Cyclic voltammetry for 7,

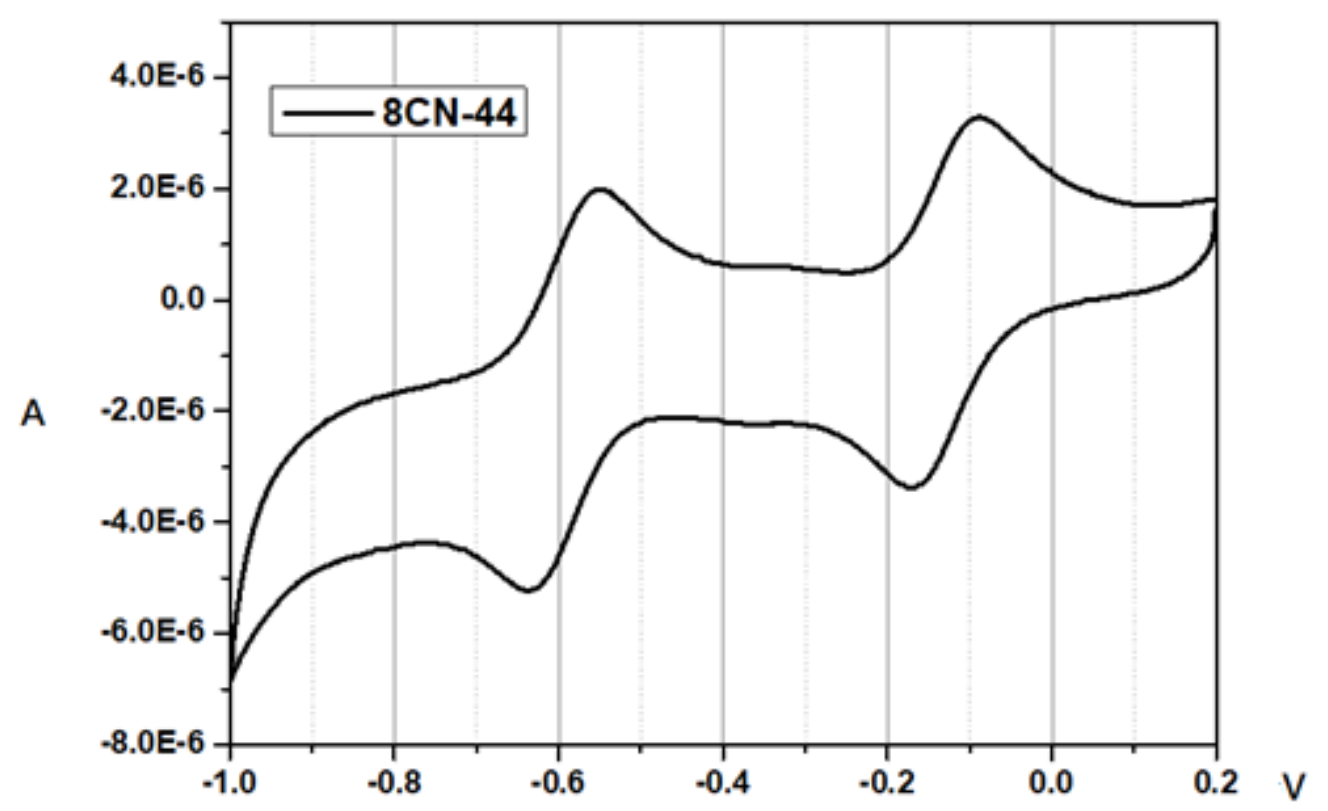

Figure 9 (SI1): Cyclic voltammetry for 8 


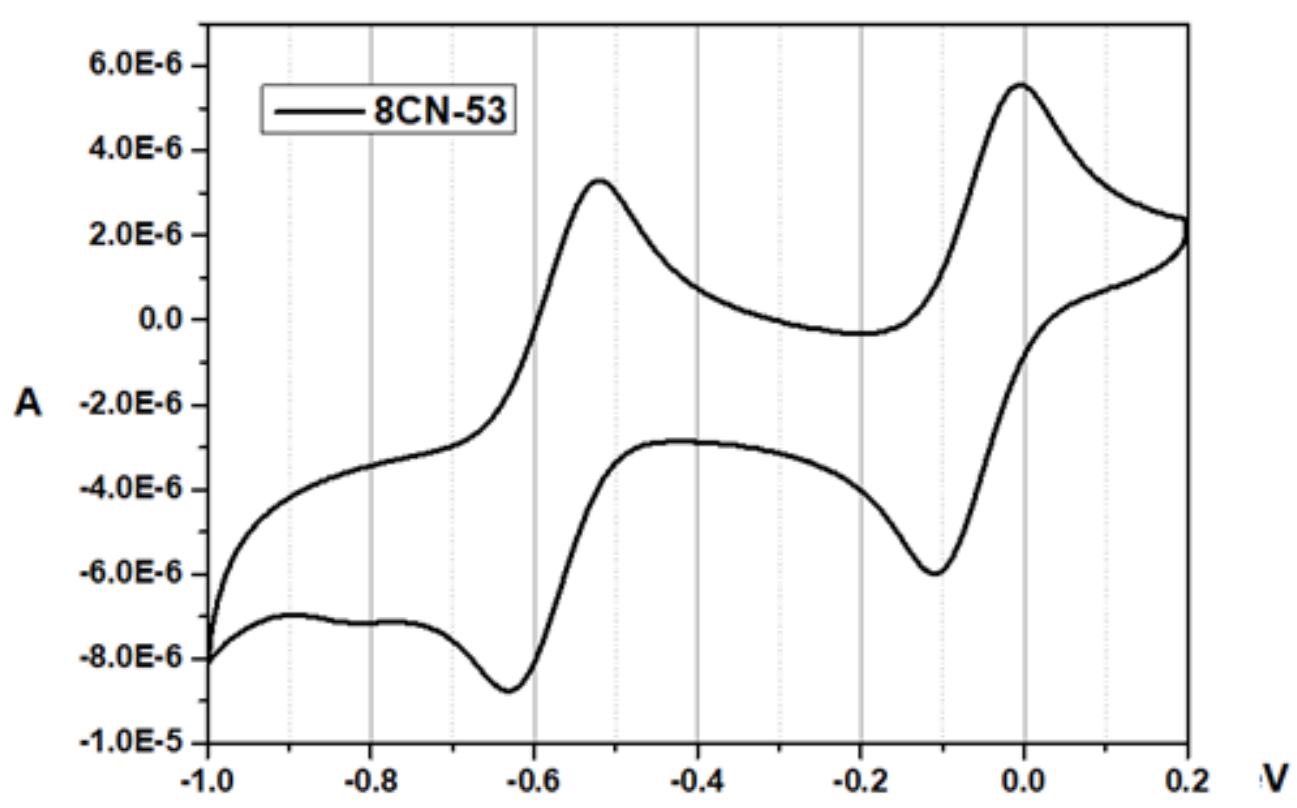

Figure 10 (SI1): Cyclic voltammetry for 9

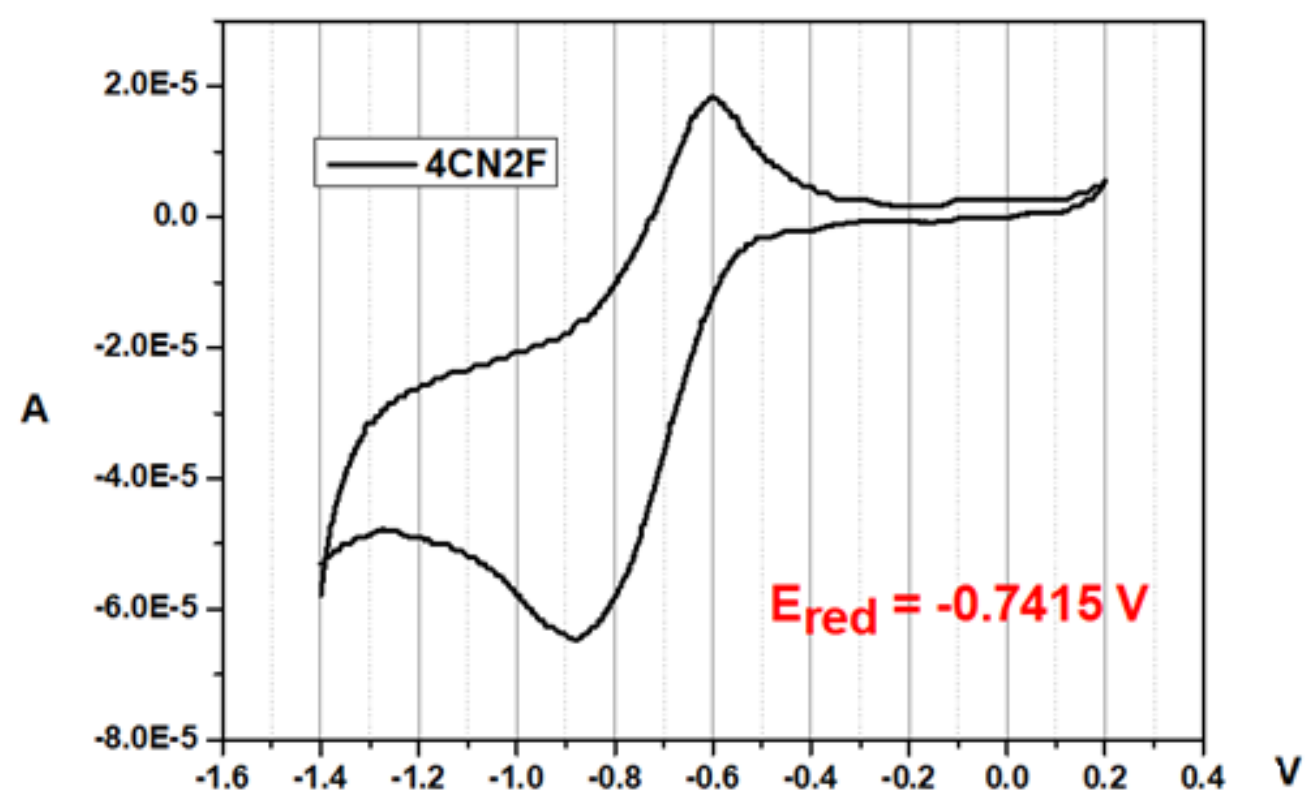

Figure 11 (SI1): Cyclic voltammetry for 14 


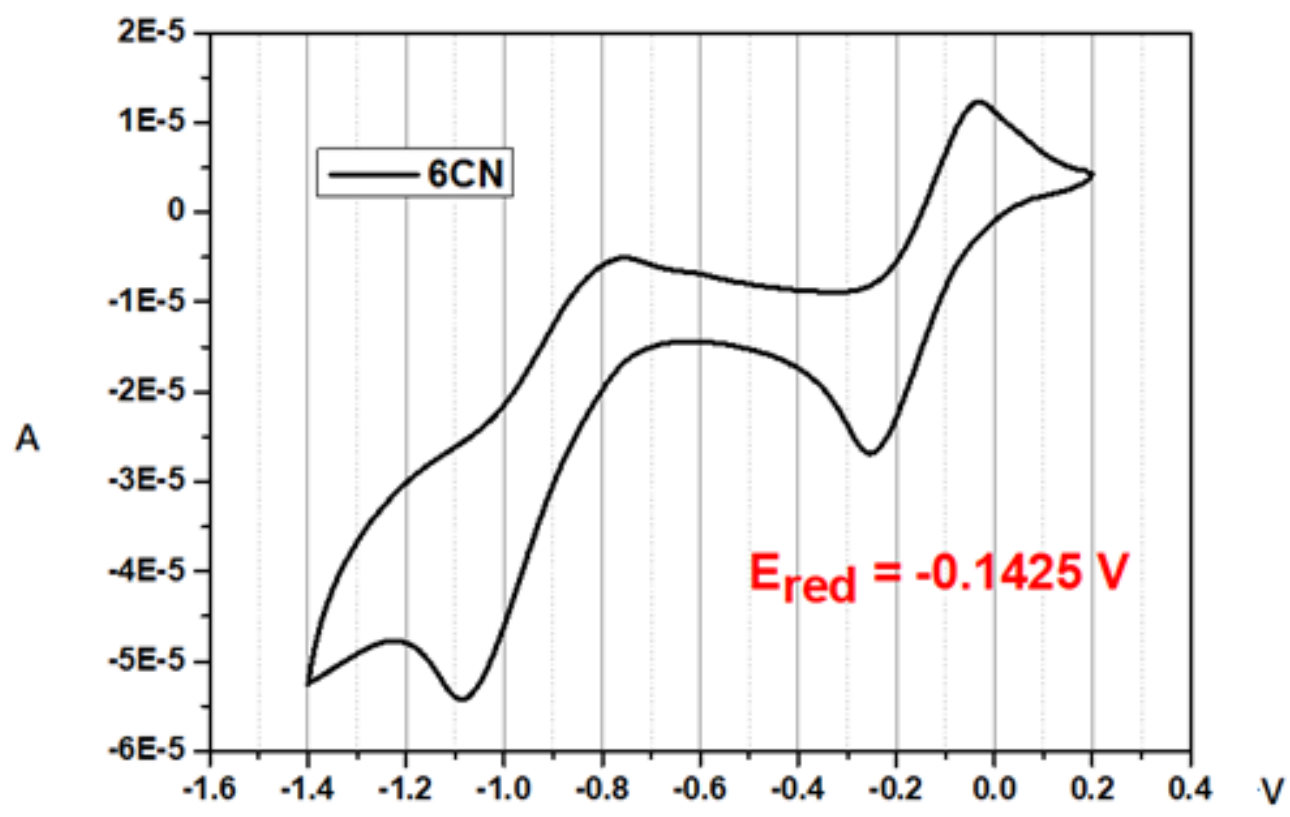

Figure 12 (SI1): Cyclic voltammetry for 15

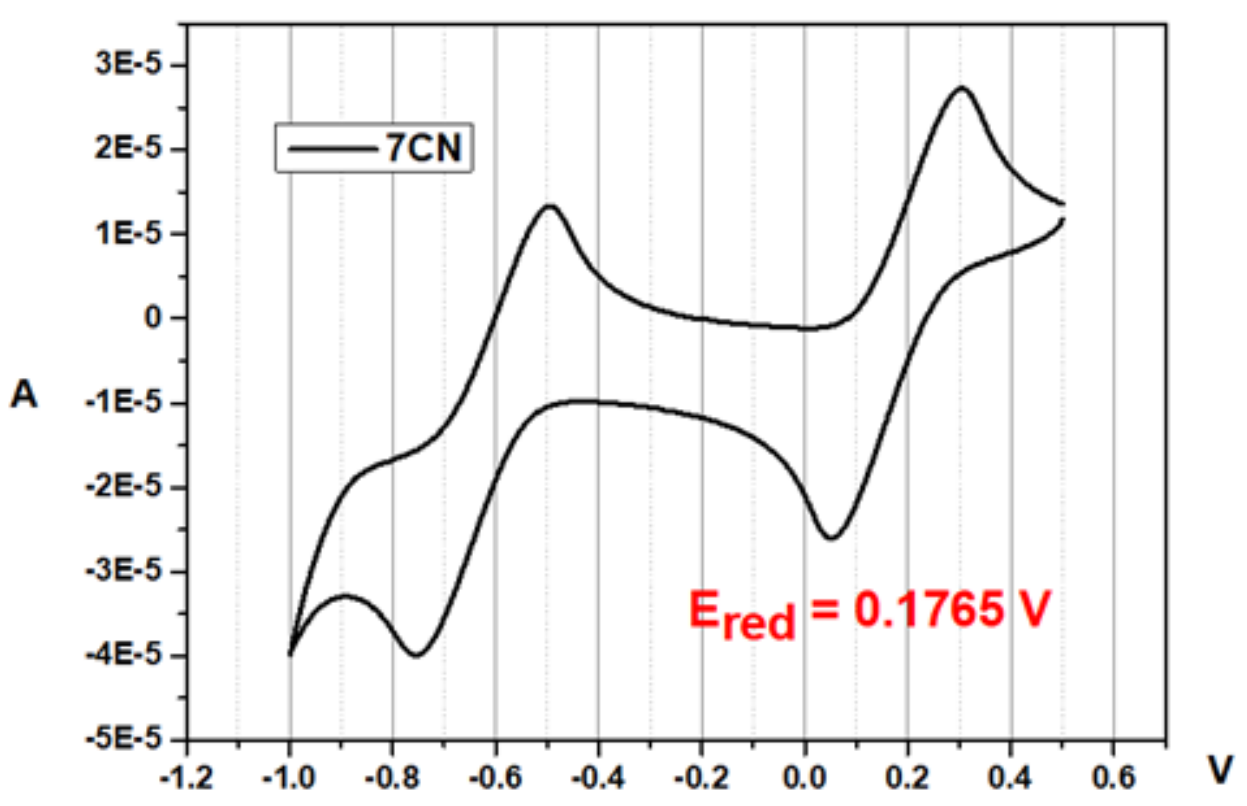

Figure 13 (SI1): Cyclic voltammetry for 16 


\begin{tabular}{|c|c|}
\hline Ferrocene reference & $\begin{array}{c}E_{o x}=0.2305(\text { for } 5-9) \\
\text { Eox }=0.489(\text { for } 14-16)\end{array}$ \\
\hline $5(4 \mathrm{CN})$ & $E_{\text {red }}=-1.106$ \\
\hline $6(6 \mathrm{CN})$ & $E_{\text {red }}=-0.566$ \\
\hline $7(7 \mathrm{CN})$ & $E_{\text {red }}=-0.313$ \\
\hline $7^{\prime}(7 \mathrm{CN})$ & $E_{\text {red }}=-0.297$ \\
\hline $8(8 \mathrm{CN})$ & $E_{\text {red }}=-0.130$ \\
\hline $9(8 \mathrm{CN})$ & $E_{\text {red }}=-0.0575$ \\
\hline $14(4 \mathrm{CN})$ & $E_{\text {red }}=-0.742$ \\
\hline $15(6 \mathrm{CN})$ & $E_{\text {red }}=-0.142$ \\
\hline $16(7 \mathrm{CN})$ & $\mathrm{E}_{\mathrm{red}}=0.176$ \\
\hline
\end{tabular}

Table: Cyclic voltammetry results of polynitrile electron acceptors 4-8, and 14-16 


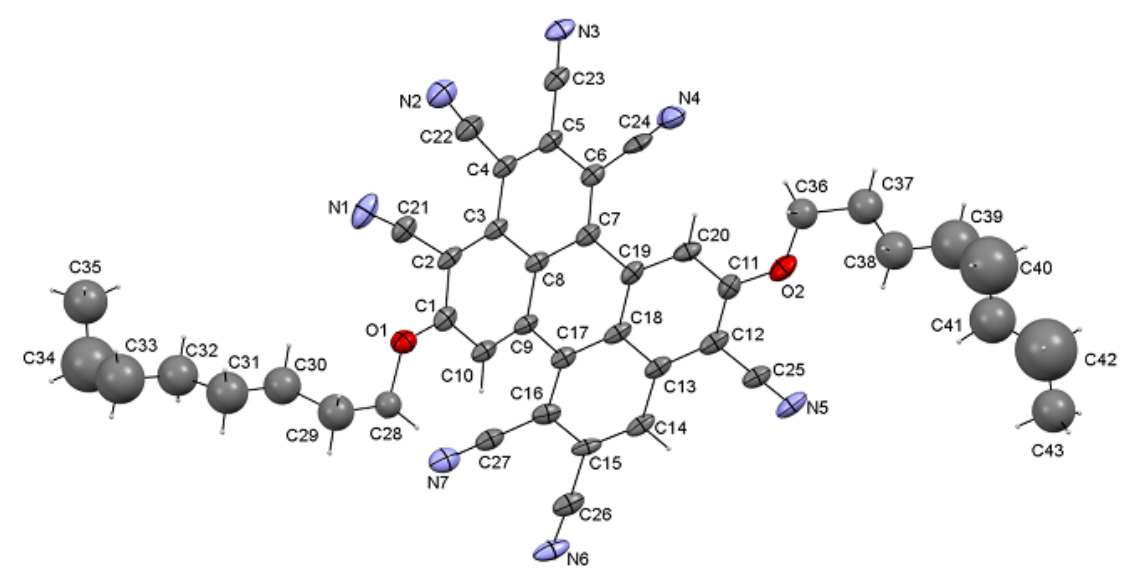

Figure 17a: ORTEP diagram of 7 (the octyl side chains are disordered)

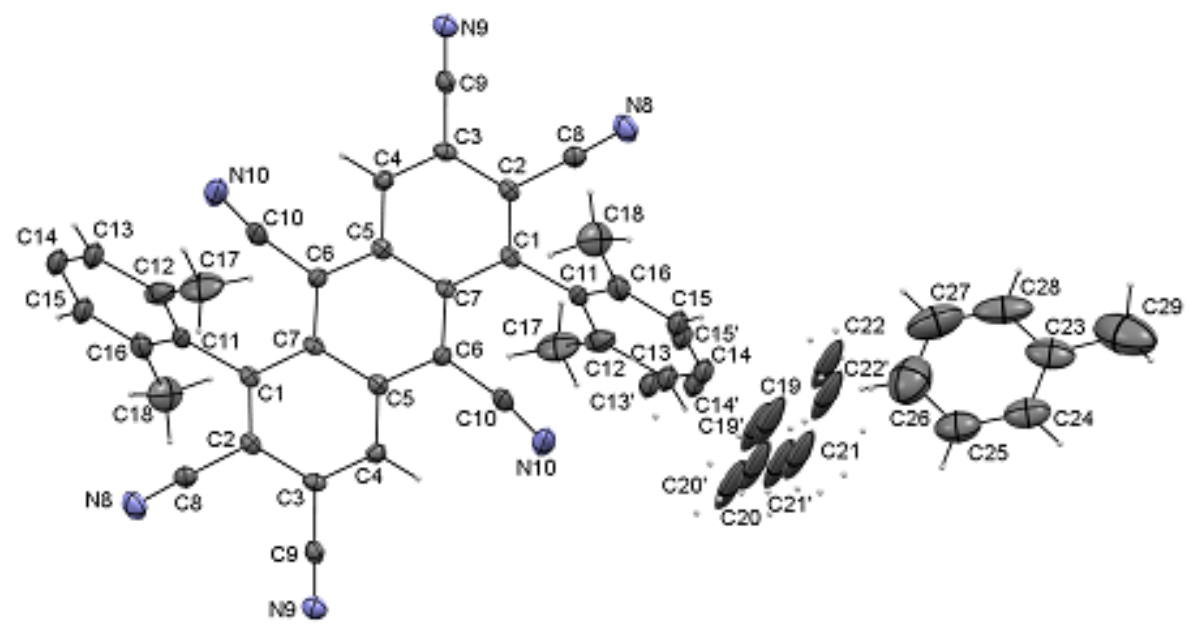

Figure 17b: ORTEP diagram of 15 with co-crystalized toluene (the t-Bu groups are disordered) 